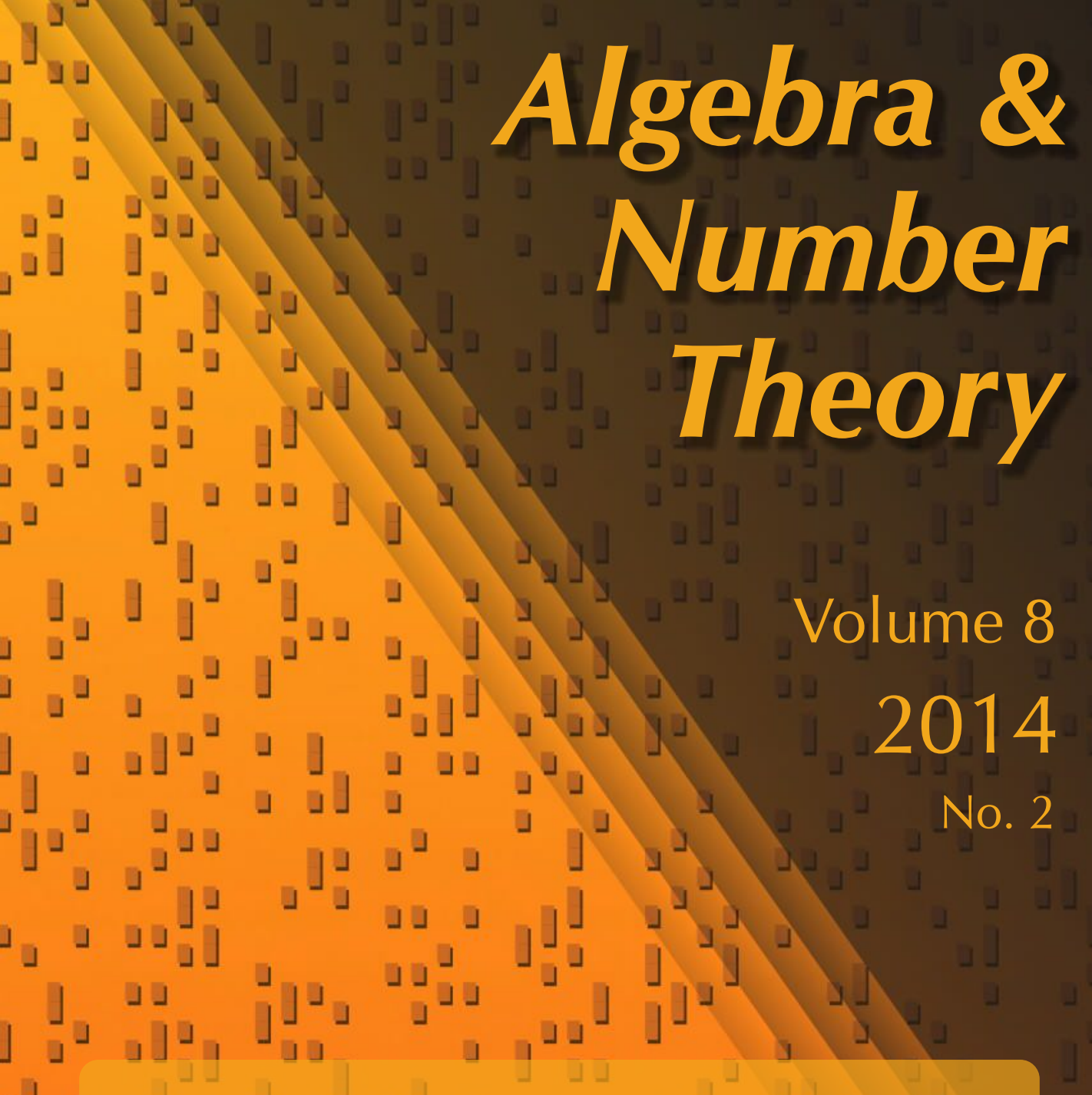

Large self-injective rings and the generating hypothesis

$\perp$ Leigh Shepperson and Neil Strickland

\lrcorner

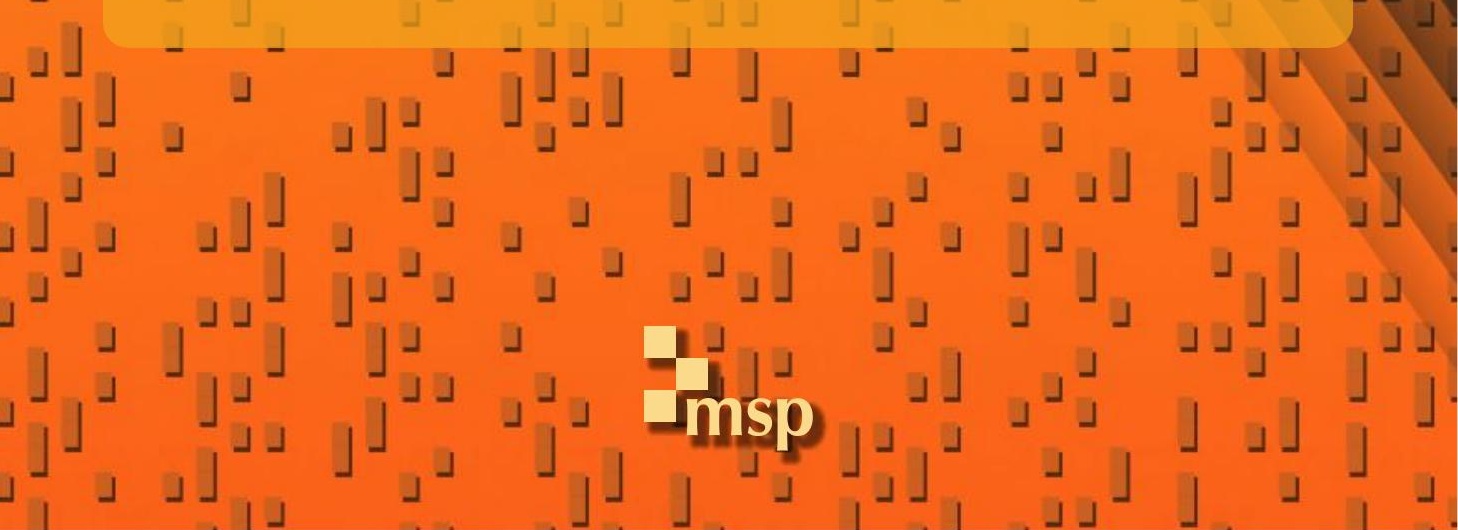




\title{
Large self-injective rings and the generating hypothesis
}

\author{
Leigh Shepperson and Neil Strickland
}

\begin{abstract}
We construct a number of different examples of non-Noetherian graded rings that are injective as modules over themselves (or have some related but weaker properties). We discuss how these are related to the theory of triangulated categories, and to Freyd's generating hypothesis in stable homotopy theory.
\end{abstract}

\section{Introduction}

In this paper we study graded commutative rings $R$ that are large in various senses (in particular, not Noetherian) and self-injective (meaning that $R$ is injective as an $R$-module). We use graded rings because they are relevant for our applications, but ungraded rings are covered as well because they can be regarded as graded rings concentrated in degree zero. The graded setting is assumed everywhere, so "element" means "homogeneous element" and "ideal" means "homogeneous ideal" and so on. Our rings will be commutative in the graded sense, so that $b a=(-1)^{|a||b|} a b$.

It is not hard to prove that any Noetherian self-injective ring is Artinian. In particular, if $R$ is a finitely generated algebra over a field $K$ that is self-injective then we must have $\operatorname{dim}_{K}(R)<\infty$ and it turns out that $R \simeq \operatorname{Hom}(R, K)$ as $R$-modules. Examples of this situation include $R=K\left[x_{1}, \ldots, x_{n}\right] /\left(r_{1}, \ldots, r_{n}\right)$ for any regular sequence $r_{1}, \ldots, r_{n}$, or the cohomology ring $R=H^{*}(M ; K)$ for any closed orientable manifold $M$. These are the most familiar examples of self-injective rings, and they are all very small. We will be looking for examples that are much larger.

Our motivation comes from a question in stable homotopy theory, which we briefly recall. In stable homotopy theory we study a certain triangulated category $\mathscr{F}$, the Spanier-Whitehead category of finite spectra. The objects can be taken to be pairs $X=(n, A)$, where $n \in \mathbb{Z}$ and $A$ is a finite simplicial complex. The morphism set $\operatorname{Hom}_{\mathscr{F}}((n, A),(m, B))$ is the set of homotopy classes of maps from $\left(\mathbb{R}^{N+n} \times A\right) \cup\{\infty\}$ to $\left(\mathbb{R}^{N+m} \times B\right) \cup\{\infty\}$, which is essentially independent of $N$ when $N$ is sufficiently large. More details are given in [Ravenel 1992], for example. For any $X, Y \in \mathscr{F}$ the set $\operatorname{Hom}_{\mathscr{F}}(X, Y)$ is a finitely generated abelian

MSC2010: primary 13C11; secondary 18E30, 55P42.

Keywords: self-injective, generating hypothesis. 
group. It turns out that most methods for studying $\operatorname{Hom}_{\mathscr{F}}(X, Y)$ treat the $p$-primary parts separately for different primes $p$. We will thus fix a prime $p$ and define $[X, Y]=\mathbb{Z}_{p} \otimes \operatorname{Hom}_{\mathscr{F}}(X, Y)$, where $\mathbb{Z}_{p}$ is the ring of $p$-adic integers. These are the morphism sets in a new triangulated category which we call $\mathscr{F}_{p}$. This has a canonical tensor structure, with the tensor product of $X$ and $Y$ written as $X \wedge Y$. The unit for this structure is called $S$, so $S \wedge X \simeq X$. As part of the triangulated structure we have a suspension functor $\Sigma: \mathscr{F}_{p} \rightarrow \mathscr{F}_{p}$, and we write $S^{n}$ for $\Sigma^{n} S$. We put $R_{n}=\left[S^{n}, S\right]$. These sets form a graded commutative ring, whose structure is extremely intricate. A great deal of partial information is known, but it seems clear that there will never be a usable complete description. Some highlights are as follows.

- $R_{n}=0$ for $n<0$, and $R_{0}=\mathbb{Z}_{p}$, and $R_{n}$ is a finite abelian $p$-group for $n>0$.

- Both the ranks and the exponents of the groups $R_{n}$ can be arbitrarily large.

- All elements in $R_{n}$ with $n>0$ are nilpotent. Thus, the reduced quotient is $R / \sqrt{0}=\mathbb{Z}_{p}$.

- Various results are available describing most or all of the structure of $R_{n}$ for $n<f(p)$, where $f(x)$ is a polynomial of degree at most three. The simplest of these says that $R_{n}=0$ for $0<n<2 p-3$, and $R_{2 p-3}=\mathbb{Z} / p$.

Now consider an arbitrary object $X \in \mathscr{F}_{p}$. We define $\pi_{n}(X)=\left[S^{n}, X\right]$ for all $n \in \mathbb{Z}$. This defines a graded abelian group $\pi_{*}(X)$, which has a natural structure as an $R$-module.

Conjecture 1.1 (Freyd's generating hypothesis). The functor $\pi_{*}: \mathscr{F}_{p} \rightarrow \operatorname{Mod}_{R}$ is faithful.

This is actually a technical modification of Freyd's conjecture [1966], because Freyd did not tensor with the $p$-adics. This causes various troubles in the development of the theory, which Freyd avoided in ad hoc ways. Much later, Hovey [2007] redeveloped the theory in the $p$-adic setting, which involves only minor modifications to Freyd's arguments but works much more smoothly.

Nearly half a century after Freyd made his conjecture, there is still no hint of a proof or a counterexample. However, there has been a certain amount of indirect progress; for example, various authors have settled the analogous questions in other triangulated categories where computations are easier [Carlson et al. 2009; Hovey et al. 2007; Benson et al. 2007; Lockridge 2007].

On the other hand, it is known that the generating hypothesis would have some very strong and surprising consequences, as we now explain.

Definition 1.2. (a) A graded ring $R$ is coherent if every finitely generated ideal is finitely presented. 
(b) A graded ring $R$ is totally incoherent if the only finitely presented ideals are 0 and $R$.

Theorem 1.3 [Freyd 1966; Hovey 2007]. Suppose that the generating hypothesis is true.

(a) The functor $\pi_{*}: \mathscr{F}_{p} \rightarrow \operatorname{Mod}_{R}$ is automatically full as well as being faithful, so it is an embedding of categories.

(b) For every object $X \in \mathscr{F}_{p}$, the image $\pi_{*}(X)$ is an injective $R$-module. In particular (by taking $X=S$ ) the ring $R$ is self-injective.

(c) The ring $R$ is totally incoherent.

Note in particular that (a) gives a full subcategory of $\operatorname{Mod}_{R}$ that has a natural triangulation. This is very unusual; in almost all known triangulated categories, the morphisms are equivalence classes of homomorphisms under some nontrivial equivalence relation, and this equivalence structure is tightly connected to the definition of the triangulation.

Our aim in this paper is to shed light on the generating hypothesis by finding examples of self-injective rings that share some of the known or conjectured properties of the stable homotopy ring $R$.

Our main results are as follows. Firstly, one cannot disprove self-injectivity by looking only in a finite range of degrees:

Theorem 1.4. Let $R$ be a graded-commutative ring such that

(a) $R_{k}=0$ for $k<0$,

(b) $R_{0}=\mathbb{Z} / 2$,

(c) $R_{k}$ is finite for all $k \geq 0$.

Suppose given $N>0$. Then there is an injective map $\phi: R \rightarrow R^{\prime}$ of graded rings such that

(1) $R^{\prime}$ also has properties (a)-(c),

(2) $\phi: R_{k} \rightarrow R_{k}^{\prime}$ is an isomorphism for $k<N$,

(3) $R^{\prime}$ is self-injective.

This result was a great surprise to the authors at least, although the proof is not too hard. We will restate and prove it as Theorem 6.6. We conjecture that the theorem remains true if we allow $R_{0}$ to be $\mathbb{Z}_{p}$, but we have not proved this.

Most of our remaining results relate to specific examples. We have aimed to give a wide spread of examples, rather than formulating each example with maximum possible generality. We will write $\mathbb{F}$ for $\mathbb{Z} / 2$. 
One of the simplest examples of a finite-dimensional self-injective ring is the exterior algebra

$$
\mathbb{F}\left[x_{0}, \ldots, x_{n}\right] /\left(x_{0}^{2}, \ldots, x_{n}^{2}\right) .
$$

Our first infinite-dimensional example is just an obvious generalisation of this.

Proposition 1.5. Let $E$ be the exterior algebra over $\mathbb{F}$ with a generator $x_{i} \in E_{2^{i}}$ for all $i \in \mathbb{N}$. Then $E$ is self-injective and coherent. The reduced quotient is $E / \sqrt{0}=\mathbb{F}$.

Self-injectivity is proved by combining Corollary 3.7 and Proposition 4.6, as will be explained in Example 4.7. The same ingredients cover many other examples, but we will not give the relevant definitions in this introduction. Coherence is proved in Proposition 5.4, and the reduced quotient is clear. We have chosen the degrees of the generators for compatibility with our other examples, but in fact the statement would remain valid if we merely assumed that $\left|x_{i}\right| \rightarrow \infty$ as $i \rightarrow \infty$.

Our next example arose by applying Theorem 1.4 to the ring $\mathbb{E}[x, y] / x y$ and studying the result in low dimensions. The result is very complicated and irregular, but after studying various recurring patterns and key features we were led to the definition below.

Theorem 1.6. Consider the ring

$$
C=\mathbb{F}\left[y_{0}, y_{1}, \ldots\right] /\left(y_{i}^{3}+y_{i} y_{i+1} \mid i \geq 0\right),
$$

with the grading given by $\left|y_{i}\right|=2^{i}$. Then $C$ is self-injective and coherent. The reduced quotient is

$$
C / \sqrt{0}=\mathbb{F}\left[x_{0}, x_{1}, \ldots\right] /\left(x_{i} x_{j} \mid i \neq j\right)=\mathbb{F} \oplus \bigoplus_{n>0} x_{n} \mathbb{F}\left[x_{n}\right],
$$

where $x_{n}=\sum_{i=0}^{n} y_{n-i}^{2^{i}}$.

This will be proved as Propositions 7.18, 7.25 and 7.26. The statement can be generalised by adjusting the degrees and the relations slightly, but this just leads to additional bookkeeping without much extra insight, so we have omitted it. It is probably also possible to generalise in more conceptual ways, but that would be a substantial project, so we leave it for future work.

For the next example, we give an axiomatic statement and then explain a special case that is relevant in chromatic homotopy theory.

Definition 1.7. For any prime $p$, we recall that

$$
\mathbb{Z}[1 / p] / \mathbb{Z} \simeq \mathbb{Q} / \mathbb{Z}_{(p)} \simeq \mathbb{Q}_{p} / \mathbb{Z}_{p} \simeq \lim _{n \rightarrow \infty} \mathbb{Z} / p^{n} .
$$

For any module $M$ over $\mathbb{Z}_{p}$, we write $M^{\vee}=\operatorname{Hom}_{\mathbb{Z}_{p}}\left(M, \mathbb{Q}_{p} / \mathbb{Z}_{p}\right)$, and call this the Pontrjagin dual of $M$. One can check that $\mathbb{Z}_{p} \vee \simeq \mathbb{Q}_{p} / \mathbb{Z}_{p}$ and $\left(\mathbb{Q}_{p} / \mathbb{Z}_{p}\right)^{\vee} \simeq \mathbb{Z}_{p}$ and $\left(\mathbb{Z} / p^{n}\right)^{\vee} \simeq \mathbb{Z} / p^{n}$. Now consider a graded $\mathbb{Z}_{p}$-algebra $R$ with a specified 
isomorphism $\zeta: R_{d} \rightarrow \mathbb{Q}_{p} / \mathbb{Z}_{p}$ for some $d$. This gives maps $\zeta^{\#}: R_{d-k} \rightarrow R_{k}^{\vee}$ by $\zeta^{\#}(a)(b)=\zeta(a b)$. We say that $R$ is Pontrjagin self-dual if all these maps are isomorphisms.

Proposition 1.8. If $R$ is Pontrjagin self-dual, then it is self-injective.

This will be proved as Proposition 8.2.

Now fix a prime $p$, and assume that $p>2$ for simplicity. Recall that $\mathscr{F}$ denotes the Spanier-Whitehead category of finite spectra. One can construct another triangulated category $\mathscr{F}^{\prime}$, called the Bousfield localisation of $\mathscr{F}$ with respect to p-local $K$-theory. Roughly speaking this is the closest possible approximation to $\mathscr{F}$ that can be analysed using topological $K$-theory, and it is computationally much more tractable than $\mathscr{F}$ itself. Ravenel's paper [1984] is a good introduction to both the conceptual framework and specific calculations, with references to original sources. Devinatz [1990] has shown that the most obvious analogue of the generating hypothesis for $\mathscr{F}^{\prime}$ is false (his Remark 1.7), but that a related statement is true (his Theorem 1). The analogue of the stable homotopy ring for $\mathscr{F}^{\prime}$ is the ring $J$ described below.

Definition 1.9. Let $p$ be an odd prime, and define a graded ring $J$ as follows. We put $J_{0}=\mathbb{Z}_{(p)}$ and $J_{-2}=\mathbb{Q}_{p} / \mathbb{Z}_{p}$; for notational convenience we use the symbol $\eta$ for the identity map $\mathbb{Z}_{(p)} \rightarrow J_{0}$, and $\zeta$ for the identity map $J_{-2} \rightarrow \mathbb{Q}_{p} / \mathbb{Z}_{p}$. Next, for each nonzero integer $k$ there is a generator $\alpha_{k} \in J_{2(p-1) k-1}$ generating a cyclic group of order $p^{v_{p}(k)+1}$, where $v_{p}(k)$ is the $p$-adic valuation of $k$. For the product structure, we have:

- $\eta(a) \eta(b)=\eta(a b)$ and $\eta(a) \zeta^{-1}(b)=\zeta^{-1}(a b)$ and $\eta(a) \alpha_{k}=a \alpha_{k}$.

- $\zeta^{-1}(a) \zeta^{-1}(b)=0$ and $\zeta^{-1}(a) \alpha_{k}=0$ for all $k$.

- If $k>0$ we have

$$
\alpha_{k} \alpha_{-k}=-\alpha_{-k} \alpha_{k}=\zeta^{-1}\left(p^{-1-v_{p}(k)}+\mathbb{Z}_{(p)}\right) .
$$

- $\alpha_{j} \alpha_{k}=0$ whenever $j+k \neq 0$.

Theorem 1.10. The ring $\hat{J}=\mathbb{Z}_{p} \otimes J$ is Pontrjagin self-dual and therefore selfinjective. It is also totally incoherent, and the reduced quotient is $\hat{J} / \sqrt{0}=\mathbb{Z}_{p}$.

Self-duality is proved as Lemma 8.3, and incoherence as Proposition 8.7. The reduced quotient is clear.

Remark 1.11. Tensoring with $\mathbb{Z}_{p}$ here just has the effect of replacing $\mathbb{Z}_{(p)}$ in degree zero with $\mathbb{Z}_{p}$. Note that this is not the same as the $p$-completion of $J$, because $\left(\mathbb{Q}_{p} / \mathbb{Z}_{p}\right)_{p}=0$. Moreover, a derived version of $p$-completion would replace $\mathbb{Q}_{p} / \mathbb{Z}_{p}$ by a copy of $\mathbb{Z}_{p}$ shifted by one degree, which is different again. The ring $J$ itself is 
not self-injective. However, this does not account for Devinatz's example showing the failure of the generating hypothesis in $\mathscr{F}^{\prime}$; that has a deeper topological origin.

We now note that the ring $\mathbb{F}[x] / x^{N}$ is another easy example of a finite-dimensional self-injective ring. Our next example arose by trying to generalise this. An obvious possibility is to consider the ring $\bigcup_{n>0} \mathbb{F}\left[x^{1 / n}\right]$ modulo the ideal generated by $x$. Any element of this ring can be expressed as $\sum_{q} a(q) x^{q}$, for some function $a: \mathbb{Q} \cap[0,1) \rightarrow \mathbb{E}$ with finite support. However, this ring needs to be adjusted to make it self-injective. Firstly, it turns out to be better not to kill $x$ itself, but just the powers $x^{q}$ with $q>1$. Next, self-injectivity forces certain modules to be isomorphic to their double duals and thus to have strong completeness properties. To handle this, we must allow some infinite sums, or equivalently weaken the condition that $a$ has finite support. It is also convenient (but not strictly necessary) to include powers $x^{q}$ where $q$ is irrational. This leads us to the following definition.

Definition 1.12. Let $K$ be a field. For any map $a:[0,1] \rightarrow K$ we put

$$
\operatorname{supp}(a)=\{q \in[0,1] \mid a(q) \neq 0\} .
$$

We say that $a$ is an infinite root series if every nonempty subset of $\operatorname{supp}(a)$ has a smallest element (so $\operatorname{supp}(a)$ is well-ordered). We let $P$ denote the set of infinite root series, and call this the infinite root algebra.

Theorem 1.13. The formula

$$
(a b)(q)=\sum_{0 \leq r \leq q} a(r) b(q-r)
$$

gives a well-defined ring structure on $P$. With this structure, $P$ is self-injective and totally incoherent. The reduced quotient is $P / \sqrt{0}=K$.

This will be proved in Propositions 9.20 and 9.21, and Corollary 9.13.

We will also discuss two rings that are not self-injective, but have a related property that we now explain.

Definition 1.14. Let $R$ be a graded commutative ring, and let $J$ be an ideal in $R$. We put $\operatorname{ann}_{R}(J)=\{a \in R \mid a J=0\}$. It is tautological that the ideal $\operatorname{ann}_{R}^{2}(J)=\operatorname{ann}_{R}\left(\operatorname{ann}_{R}(J)\right)$ contains $J$. We say that $R$ satisfies the double annihilator condition if $\operatorname{ann}_{R}^{2}(J)=J$ for all finitely generated ideals $J$.

Proposition 1.15. If $R$ is self-injective then it satisfies the double annihilator condition. Conversely, if $R$ is Noetherian and satisfies the double annihilator condition, then it is self-injective.

This is proved in Remark 2.4 and Theorem 4.1. 
Definition 1.16. For any integer $n$ we let $B(n)$ be the set of exponents $i$ such that $2^{i}$ occurs in the binary expansion of $n$, so $B(n)$ is the unique finite subset of $\mathbb{N}$ such that $n=\sum_{i \in B(n)} 2^{i}$.

The Rado graph has vertex set $\mathbb{N}$, with an edge from $i$ to $j$ if $(i \in B(j)$ or $j \in B(i)$ ). The Rado ideal in the exterior algebra $E$ has a generator $x_{i} x_{j}$ for each pair $(i, j)$ such that there is no edge from $i$ to $j$ in the Rado graph. The Rado algebra $Q$ is the quotient of $E$ by the Rado ideal.

Remark 1.17. See [Rado 1964; Cameron 2001] for discussion of the Rado graph. Although the definition looks very specialised, the appearance is deceptive. Roughly speaking, any countable random graph is isomorphic to the Rado graph with probability one. The proof of this uses a kind of injectivity property of the Rado graph, which is what suggested it to us as being potentially relevant for the present project.

Theorem 1.18. The Rado algebra is totally incoherent (and in particular, not Noetherian). It satisfies the double annihilator condition, but is not self-injective. The reduced quotient is $Q / \sqrt{0}=\mathbb{F}$.

This will be proved as Propositions 10.5, 10.6 and 10.8 (apart from the fact that $Q / \sqrt{0}=\mathbb{F}$, which is clear).

One major difference between the Rado algebra and the stable homotopy ring is that the former has Krull dimension zero (because all elements in the maximal ideal square to zero) whereas the latter is $\mathbb{Z}_{2}$ in degree 0 and so has Krull dimension one. Our final example aims to do something similar to the Rado construction but without making all the generators nilpotent. To do this we must work in base $\omega$ rather than base 2; this involves some theory of ordinals, which we briefly recall (the book [Johnstone 1987] is an admirably concise reference). There is an exponentiation operation for ordinals (different from the usual one for cardinals). There is a countable ordinal called $\epsilon_{0}$ such that $\epsilon_{0}=\omega^{\epsilon_{0}}$, and no ordinal $\alpha<\epsilon_{0}$ satisfies $\alpha=\omega^{\alpha}$. Any ordinal $\alpha<\epsilon_{0}$ has a unique Cantor normal form

$$
\alpha=\omega^{\beta_{1}} n_{1}+\cdots+\omega^{\beta_{r}} n_{r},
$$

where the $n_{i}$ are positive integers and $\alpha>\beta_{1}>\cdots>\beta_{r}$.

Definition 1.19. We write $\mu_{0}(\alpha, \beta)$ for the coefficient of $\omega^{\beta}$ in the Cantor normal form of $\alpha$. We then put

$$
\mu(\alpha, \beta)=\max \left(\mu_{0}(\alpha, \beta), \mu_{0}(\beta, \alpha)\right),
$$

and

$$
A=\mathbb{F}\left[x_{\alpha} \mid \alpha<\epsilon_{0}\right] /\left(x_{\alpha} x_{\beta}^{1+\mu(\alpha, \beta)} \mid \alpha, \beta<\epsilon_{0}, \alpha \neq \beta\right) .
$$

We call $A$ the $\epsilon_{0}$-algebra. 
Given any function $\delta: \epsilon_{0} \rightarrow \mathbb{N}$, we can give $A$ a grading such that $\left|x_{\alpha}\right|=\delta(\alpha)$. In Section 11 we will describe a particular function $\delta$ with the property that $\delta(\alpha)>0$ for all $\alpha$, and all the sets $\delta^{-1}\{n\}$ are finite. This will ensure that the homogeneous pieces $A_{d}$ are finite for all $d$.

Theorem 1.20. If $J$ is any ideal in $A$ that is generated by a finite set of monomials, then $J=\operatorname{ann}_{A}^{2}(J)$. However, there are nonmonomial ideals $J$ with $J \neq \operatorname{ann}_{A}^{2}(J)$, so A does not satisfy the double annihilator condition, and is not self-injective. Moreover, A is totally incoherent, and the reduced quotient is

$$
A / \sqrt{0}=\mathbb{F}\left[x_{\alpha} \mid \alpha<\epsilon_{0}\right] /\left(x_{\alpha} x_{\beta} \mid \alpha \neq \beta\right) .
$$

This will be proved as Propositions 11.17, 11.21 and 11.22, and Corollary 11.19.

\section{General theory of self-injective rings}

Let $R$ be a graded commutative ring, and $\operatorname{Mod}_{R}$ the category of graded $R$-modules. Suppose that $R$ is self-injective. For $M \in \operatorname{Mod}_{R}$ we put $D M=\operatorname{Hom}_{R}(M, R)$ (regarded as a graded $R$-module in the usual way). This construction defines a functor $D: \operatorname{Mod}_{R} \rightarrow \operatorname{Mod}_{R}^{\mathrm{op}}$, which is exact because $R$ is self-injective. It follows that $D^{2}$ gives an exact covariant functor from $\operatorname{Mod}_{R}$ to itself. There is a natural map $\kappa: M \rightarrow D^{2} M$ given by $\kappa(m)(u)=u(m)$. Properties of $D^{2}$ are studied under different technical hypotheses in [Bruns and Herzog 1993, Theorem 3.2.13], for example.

Definition 2.1. We let $U=U_{R}$ denote the full subcategory of $\operatorname{Mod}_{R}$ consisting of the modules $M$ for which $\kappa: M \rightarrow D^{2} M$ is an isomorphism.

Proposition 2.2. The category $\mathcal{U}$ is closed under finite direct sums, suspensions and desuspensions, kernels, cokernels, images and extensions. It also contains $R$ itself.

Proof. This is clear from the exactness of the functor $D^{2}$ and the five lemma.

Corollary 2.3. If $J \leq R$ is a finitely generated ideal, then $J$ and $R / J$ lie in $U$.

Proof. They are the image and cokernel of some map $\bigoplus_{i=1}^{n} \Sigma^{d_{i}} R \rightarrow R$.

Remark 2.4. If $J$ is an ideal in $R$ then

$$
D(R / J) \simeq\{a \in R \mid a J=0\}=\operatorname{ann}_{R}(J) .
$$

By dualising the sequence $J \rightarrow R \rightarrow R / J$, we see that $D(J)=R / \operatorname{ann}_{R}(J)$. It follows that $D^{2}(J)=\operatorname{ann}_{R}\left(\operatorname{ann}_{R}(J)\right)=\operatorname{ann}_{R}^{2}(J)$. Thus, we have $J \in \mathcal{U}$ if and only if $J=\operatorname{ann}_{R}^{2}(J)$. In particular, if $J$ is finitely generated then $J=\operatorname{ann}_{R}^{2}(J)$.

Lemma 2.5. For any $a \in R_{d}$ there is an isomorphism $D(R a) \simeq \Sigma^{-d} R a$. 
Proof. Given $u \in D(R a)_{e}$ we put $\alpha(u)=u(a) \in R_{d+e}$. This defines a map $\alpha: D(R a) \rightarrow \Sigma^{-d} R$, which is clearly injective. Note that if $b \in \operatorname{ann}_{R}(a)$ then $\alpha(a) b=\alpha(a b)=\alpha(0)=0$. This proves that $\alpha(a) \in \operatorname{ann}_{R}^{2}(R a)_{d+e}=(R a)_{d+e}$. In the opposite direction, if $c \in(R a)_{d+e}$ then we have $c=m a$ for some $m \in R_{e}$, and the rule $\mu_{m}(x)=m x$ defines an element $\mu_{m} \in D(R a)_{e}$ with $\alpha\left(\mu_{m}\right)=c$. This proves that the image of $\alpha$ is $\Sigma^{-d} R a$, as required.

Proposition 2.6. If $R$ is self-injective and $a \in R$ then $R / \operatorname{ann}(a)$ is also selfinjective.

Proof. Put $Q=R / \operatorname{ann}(a)$, and let $i: Q \rightarrow R$ be induced by $x \mapsto x a$, so $i$ is injective, with image $R a$. For $M \in \operatorname{Mod}_{Q}$ we write

$$
D_{Q}(M)=\operatorname{Hom}_{Q}(M, Q)=\operatorname{Hom}_{R}(M, Q) \quad \text { and } \quad D_{R}(M)=\operatorname{Hom}_{R}(M, R) .
$$

We are given that $D_{R}$ is exact, and we must show that $D_{Q}$ is exact. The map $i: Q \rightarrow R$ gives a natural monomorphism $i: D_{Q}(M) \rightarrow D_{R}(M)$, and it will suffice to show that this is also an epimorphism. For any $\phi: M \rightarrow R$ we see that $\operatorname{ann}(a) . \phi(M)=\phi(\operatorname{ann}(a) M)=\phi(0)=0$, so $\phi(M) \leq \operatorname{ann}_{R}^{2}(a)=R a$, and $i: Q \rightarrow R a$ is an isomorphism, so $\phi=i(\psi)$ for some $\psi \in D_{Q}(M)$, as required.

Proposition 2.7. If $R$ is self-injective and $I$ and $J$ are ideals in $R$, then

$$
\operatorname{ann}_{R}(I+J)=\operatorname{ann}_{R}(I) \cap \operatorname{ann}_{R}(J) \quad \text { and } \quad \operatorname{ann}_{R}(I \cap J)=\operatorname{ann}_{R}(I)+\operatorname{ann}_{R}(J) .
$$

Proof. There is a short exact sequence

$$
R /(I \cap J) \stackrel{\left[\begin{array}{l}
1 \\
1
\end{array}\right]}{\longrightarrow} R / I \oplus R / J \stackrel{[1-1]}{\longrightarrow} R /(I+J) .
$$

By applying the exact functor $D$, we get a short exact sequence

$$
\operatorname{ann}_{R}(I \cap J) \stackrel{[1}{\longleftarrow} \operatorname{ann}_{R}(I) \oplus \operatorname{ann}_{R}(J) \stackrel{\left[\begin{array}{c}
1 \\
-1
\end{array}\right]}{\longleftarrow} \operatorname{ann}_{R}(I+J) .
$$

The claim follows.

Corollary 2.8. If $R$ is local and self-injective and $I$ and $J$ are nontrivial ideals, then $I \cap J$ is also nontrivial.

Proof. Let $\mathfrak{m}$ be the maximal ideal. As $I$ and $J$ are nontrivial we have $\operatorname{ann}(I)<R$ and $\operatorname{ann}(J)<R$, so ann $(I) \leq \mathfrak{m}$ and $\operatorname{ann}(J) \leq \mathfrak{m}$, so

$$
\operatorname{ann}(I \cap J)=\operatorname{ann}(I)+\operatorname{ann}(J) \leq \mathfrak{m}<R,
$$

so $I \cap J$ is nontrivial. 


\section{Criteria for self-injectivity}

We first record a graded version of the standard Baer criterion for injectivity.

Definition 3.1. Let $R$ be a graded ring, and let $I$ be a graded $R$-module. We say that $I$ satisfies the Baer condition if for every graded ideal $J \leq R$, every integer $d$ and every $R$-module homomorphism $\phi: \Sigma^{d} J \rightarrow I$, there exists $m \in I_{d}$ such that $\phi(a)=a m$ for all $a \in I$. We say that $I$ satisfies the finite Baer condition if the same condition holds for all finitely generated graded ideals $J$.

Proposition 3.2. In the above context, the module I is injective if and only if it satisfies the Baer condition.

Proof. This was originally done in the ungraded context in [Baer 1940], as an application of Zorn's lemma. The proof is also given in many textbooks, such as [Lam 1999, page 63]. It can be modified in an obvious way to keep track of gradings, which gives our statement above.

Proposition 3.3. Suppose that $I_{d}$ is finite for all $d$, and that I satisfies the finite Baer condition. Then I also satisfies the full Baer condition and so is injective.

Proof. Consider a graded ideal $J \leq R$ and a homomorphism $\phi: \Sigma^{d} J \rightarrow I$. For each finitely generated ideal $K \subseteq J$ we put

$$
M(K)=\left\{m \in I_{d} \mid \phi(a)=a m \text { for all } a \in K\right\} .
$$

The finite Baer condition means that this is a nonempty subset of the finite set $I_{d}$. Choose $K$ such that $|M(K)|$ is as small as possible, and choose $m \in M(K)$. For $a \in J$ it is clear that $M(K+R a) \subseteq M(K)$, so by the minimality property we must have $M(K+R a)=M(K)$, so $m \in M(K+R a)$, so $\phi(a)=a m$. This proves the full Baer condition.

Definition 3.4. Let $R$ be a graded ring, and let $I$ be an $R$-module. A test pair of length $r$ and degree $d$ is a pair $(u, v)$ where $u \in R^{r}$ and $v \in I^{r}$ such that the entries $u_{i}$ and $v_{i}$ are homogeneous with $\left|v_{i}\right|=\left|u_{i}\right|+d$ for all $i$. A block for such a pair is a vector $b \in R^{r}$ such that $b . u=0$ but $b . v \neq 0$ (where $b . x=\sum_{i} b_{i} x_{i}$ ). A transporter is an element $m \in I_{d}$ such that $v_{i}=m u_{i}$ for all $i$.

Remark 3.5. We implicitly formulate the theory of graded groups in such a way that the zero elements in different degrees are distinct. Thus, the notation $|u|$ is meaningful even if $u=0$.

Proposition 3.6. The module I satisfies the finite Baer condition if and only if every test pair has either a block or a transporter.

Proof. Suppose that every test pair has either a block or a transporter. Consider a finitely generated graded ideal $J \leq R$, and a homomorphism $\phi: \Sigma^{d} J \rightarrow R$. 
Choose a list $u=\left(u_{1}, \ldots, u_{r}\right)$ of homogeneous elements that generates $J$, and put $v_{i}=\phi\left(u_{i}\right) \in I$. Note that if $b \in R^{r}$ with $b . u=0$ then we can apply $\phi$ to see that $b \cdot v=0$. It follows that the pair $(u, v)$ has no block, so it must have a transporter. This means that there is an element $m \in I_{d}$ with $\phi\left(u_{i}\right)=u_{i} m$ for all $i$, and it follows easily that $\phi(a)=a m$ for all $a \in J$, as required.

Conversely, suppose that $I$ satisfies the finite Baer condition. Consider a test pair $(u, v)$ of degree $d$ with no block, and let $J$ be the ideal generated by the entries $u_{i}$. Define $\phi: \Sigma^{d} J \rightarrow I$ by $\phi\left(\sum_{i} b_{i} u_{i}\right)=\sum_{i} b_{i} v_{i}$ (the absence of a block means that this is well-defined). The finite Baer condition means that there is an element $m \in I_{d}$ with $\phi(a)=a m$ for all $a \in J$, and this $m$ is clearly a transporter for $(u, v)$.

Corollary 3.7. Let $R$ be a graded commutative ring such that $R_{k}$ is finite for all $k$. Suppose also that there are subrings

$$
R(0) \leq R(1) \leq R(2) \leq \cdots \leq R
$$

such that each $R(n)$ is self-injective and $R=\bigcup_{n} R(n)$. Then $R$ is self-injective.

Proof. Any test pair $(u, v) \in R^{r} \times R^{r}$ can be regarded as a test pair over $R(n)$ for sufficiently large $n$. As $R(n)$ is self-injective, there must be a block in $R(n)^{r}$ or a transporter in $R(n)$. It is clear from the definitions that such a block or transporter still qualifies as a block or transporter over $R$, so we see that $R$ satisfies the finite Baer condition. As we have assumed that $R_{k}$ is finite for all $k$, we can use Proposition 3.3 to see that $R$ is injective as an $R$-module.

Theorem 3.8. Let $R$ be a graded commutative ring such that $R_{k}$ is finite for all $k$. The following conditions are equivalent:

(a) $R$ is self-injective.

(b) For all finitely generated ideals $J, K \leq R$ we have $\operatorname{ann}_{R}^{2}(J)=J$ and

$$
\operatorname{ann}_{R}(J \cap K)=\operatorname{ann}_{R}(J)+\operatorname{ann}_{R}(K) .
$$

(c) For all elements $a \in R$ and every finitely generated ideal $J \leq R$ we have $\operatorname{ann}_{R}^{2}(a)=R a$ and

$$
\operatorname{ann}_{R}(J \cap R a)=\operatorname{ann}_{R}(J)+\operatorname{ann}_{R}(a) .
$$

Proof. It follows from Remark 2.4 and Proposition 2.7 that (a) implies (b). If (b) holds, then (c) follows immediately. Now suppose (c) holds. As we have assumed that $R_{k}$ is finite for all $k$, we may use the theory of blocks and transporters. We proceed by induction on the length of a test pair to show that every test pair over the ring $R$ has either a block or a transporter. Let $(u ; v)$ be a test pair of length 1 and degree $d$. Suppose this test pair has neither block nor transporter. Then $\operatorname{ann}_{R}(u) \leq \operatorname{ann}_{R}(v)$ and by assumption we have $R v=\operatorname{ann}_{R}^{2}(v) \leq \operatorname{ann}_{R}^{2}(u)=R u$, 
that is, $v=u m$ for some $m \in R_{d}$. Since $m$ is a transporter for this test pair, we have a contradiction.

Now suppose each test pair of length $\leq k$ and arbitrary degree has either a block or a transporter. A test pair of length $k+1$ and degree $d$ takes the form $\left(u, u_{k+1} ; v, v_{k+1}\right)$, where $(u ; v)$ is a test pair of length $k$ and degree $d$ and $\left(u_{k+1}, v_{k+1}\right)$ is a test pair of length 1 and degree $d$. By the inductive hypothesis, both the test pairs $(u ; v)$ and $\left(u_{k+1}, v_{k+1}\right)$ have either a block or a transporter. If $(u ; v)$ has block $r$, then $(r, 0)$ is a block for the test pair $\left(u, u_{k+1} ; v, v_{k+1}\right)$. Similarly, if $\left(u_{k+1}, v_{k+1}\right)$ has block $r_{k+1}$, then $\left(0, \ldots, 0, r_{k+1}\right)$ is a block for the test pair $\left(u, u_{k+1} ; v, v_{k+1}\right)$. Otherwise, $(u ; v)$ must have transporter $m \in R_{d}$ and $\left(u_{k+1}, v_{k+1}\right)$ must have transporter $n \in R_{d}$. In this situation, suppose the test pair $\left(u, u_{k+1} ; v, v_{k+1}\right)$ has neither block nor transporter and let $J$ be the ideal generated by the entries of $u$. The absence of a block implies that there is a well defined map $\phi: \Sigma^{d}\left(J+R u_{k+1}\right) \rightarrow R$ defined by $\phi\left(\sum_{i=1}^{k+1} b_{i} u_{i}\right)=\sum_{i=1}^{k+1} b_{i} v_{i}$. Now let $s$ be an element in the intersection $J \cap R u_{k+1}$. Then we must have $s=\sum_{i=1}^{k} s_{i} u_{i}=s_{k+1} u_{k+1}$ for elements $s_{i} \in R$ for each $i$. Applying the map $\phi$ to the zero element $\left(\sum_{i=1}^{k} s_{i} u_{i}\right)-s_{k+1} u_{k+1}$ gives

$$
0=\left(\sum_{i=1}^{k} s_{i} v_{i}\right)-s_{k+1} v_{k+1}=\left(\sum_{i=1}^{k} s_{i} u_{i} m\right)-s_{k+1} u_{k+1} n=s(m-n) .
$$

Thus it follows that the element $m-n$ is in the annihilator ideal $\operatorname{ann}_{R}\left(J \cap R u_{k+1}\right)$. By assumption, we have $\operatorname{ann}_{R}\left(J \cap R u_{k+1}\right)=\operatorname{ann}_{R}(J)+\operatorname{ann}_{R}\left(u_{k+1}\right)$. Now let $m-n=x-y$, where $x \in \operatorname{ann}_{R}(J)$ and $y \in \operatorname{ann}_{R}\left(u_{k+1}\right)$, and put $z=m-x=n-y$. Since $u_{i} z=u_{i}(m-x)=u_{i} m=v_{i}$ for each $i \leq k$ and $u_{k+1} z=u_{k+1}(n-y)=$ $u_{k+1} n=v_{k+1}$ it follows that $z$ is a transporter for the test pair $\left(u, u_{k+1} ; v, v_{k+1}\right)$. As this gives a contradiction, it follows that every test pair of length $k+1$ and arbitrary degree must have either a block or transporter. We deduce that every test pair in the ring $R$ must have either a block or transporter, and since $R_{k}$ is finite for each $k$, we can use Proposition 3.6 to show that $R$ is injective as an $R$-module.

\section{The Noetherian case}

Theorem 4.1. Let $R$ be a Noetherian graded commutative ring. Then the following are equivalent:

(a) $R$ is self-injective.

(b) For every ideal $J \leq R$ we have $\operatorname{ann}_{R}^{2}(J)=J$.

(c) $R$ is Artinian (and thus is a finite product of Artinian local rings), and each of the local factors has one-dimensional socle. 
Statements similar to this are certainly well-known (see, for example, [Bruns and Herzog 1993, Exercise 3.2.15]), but we do not know a reference for this precise formulation. For completeness we will give a self-contained proof after some lemmas.

Lemma 4.2. Let $R$ be an Artinian local graded ring, with maximal ideal $\mathfrak{m}$, and put $K=R / \mathfrak{m}$. Suppose that the socle $\operatorname{soc}(R)=\operatorname{ann}_{R}(\mathfrak{m})$ has dimension one over $K$. Then every nonzero ideal in $R$ contains $\operatorname{soc}(R)$.

Proof. Let $I$ be a nonzero ideal. By the Artinian condition, we can choose an ideal $J$ that is minimal among nonzero ideals contained in $I$. Recall that every Artinian ring is Noetherian (see, for example, [Matsumura 1980, Theorem 3.2]), so we can use Nakayama's lemma to see that $\mathfrak{m} J<J$ and thus (by minimality) that $\mathfrak{m} J=0$. This means that $J$ is a nontrivial $K$-subspace of $\operatorname{soc}(R)$, but $\operatorname{soc}(R)$ has dimension one, so $J=\operatorname{soc}(R)$, so $\operatorname{soc}(R) \leq I$.

Lemma 4.3. Suppose that $R$ is as in Lemma 4.2. Then for all ideals $J \leq R$ we have $\operatorname{ann}_{R}^{2}(J)=J$.

Proof. First, it is standard that we can fit together a composition series for $J$ with a composition series for $R / J$ to get a chain

$$
0=I_{0}<I_{1}<\cdots<I_{r}=R
$$

with $I_{i} / I_{i-1} \simeq K$ for all $i$, and $J=I_{t}$ for some $t$. Now let $A_{j}$ be the annihilator of $I_{j}$, so we have

$$
R=A_{0} \geq A_{1} \geq \cdots \geq A_{r}=0 .
$$

Now $\mathfrak{m} A_{i} I_{i+1}=A_{i}\left(\mathfrak{m} I_{i+1}\right) \leq A_{i} I_{i}=0$, so $A_{i} I_{i+1} \leq \operatorname{soc}(R)$. On the other hand, we have $A_{i} I_{i}=0$ and $A_{i+1} I_{i+1}=0$. We therefore have a natural map

$$
\xi_{i}: A_{i} / A_{i+1} \rightarrow \operatorname{Hom}_{K}\left(I_{i+1} / I_{i}, \operatorname{soc}(R)\right)
$$

given by $\xi_{i}\left(a+A_{i+1}\right)\left(b+I_{i}\right)=a b$. It is clear from the definitions that this is injective, and the codomain is isomorphic to $K$, so $A_{i} / A_{i+1}$ is either 0 or $K$. It is standard that any two composition series have the same length, so we must have $A_{i} / A_{i+1} \simeq K$ for all $i$, so $A_{i}$ has length $r-i$. After applying the same logic to the composition series $\left\{A_{r-i}\right\}_{i=0}^{r}$ we see that the ideal ann $\left(A_{i}\right)=\operatorname{ann}^{2}\left(I_{i}\right)$ has length $i$. We also know that $I_{i} \leq \operatorname{ann}^{2}\left(I_{i}\right)$ and that $I_{i}$ also has length $i$; it follows that $I_{i}=\operatorname{ann}^{2}\left(I_{i}\right)$, as required.

Corollary 4.4. Suppose that $R$ is as in Lemma 4.3. Then $R$ is self-injective.

Proof. Consider an ideal $I \leq R$ and an $R$-module map $f: I \rightarrow R$. Choose a composition series $0=J_{0}<J_{1}<\cdots<J_{r}=I$. We have $J_{i} / J_{i-1} \simeq K$ so we can find $a_{i} \in J_{i} \backslash J_{i-1}$ such that $J_{i}=J_{i-1}+R a_{i}$ with $\mathfrak{m} a_{i} \leq J_{i-1}$. 
We will construct elements $x_{0}, \ldots, x_{r} \in R$ such that $f(a)=a x_{i}$ for all $a \in J_{i}$. We start with $x_{0}=0$. Now suppose we have found $x_{i-1}$. Put $u_{i}=f\left(a_{i}\right)-x_{i-1} a_{i}$. Using the fact that $\mathfrak{m} a_{i} \leq I_{i-1}$ we find that $\mathfrak{m} u_{i}=0$, so $u_{i} \in \operatorname{soc}(R)$. Next, we have $a_{i} \notin I_{i-1}=\operatorname{ann}^{2}\left(I_{i-1}\right)$, so $\operatorname{ann}\left(I_{i-1}\right) a_{i} \neq 0$. As every nontrivial ideal contains the socle, we see that $u_{i} \in \operatorname{ann}\left(I_{i-1}\right) a_{i}$, so we can write $u_{i}=y_{i} a_{i}$ for some $y_{i}$ with $y_{i} I_{i-1}=0$. We now put $x_{i}=x_{i-1}+y_{i}$. By construction we have $f(a)=a x_{i}$ for $a \in I_{i-1}$ or for $a=a_{i}$, and it follows that this equation holds for all $a \in I_{i}$ as required. At the end of the induction we have an element $x_{r}$ which fulfils Baer's criterion.

Proof of Theorem 4.1. It follows from Remark 2.4 that (a) implies (b). Now suppose that (b) holds. Consider a descending chain of ideals $I_{0} \geq I_{1} \geq I_{2} \geq \cdots$ in $R$. The ideals ann $\left(I_{k}\right)$ then form an ascending chain, which must eventually stabilise because $R$ is Noetherian. We can thus take annihilators again to see that the original chain also stabilises. This shows that $R$ is Artinian. It follows in a standard way that there are only finitely many maximal ideals, and that $R$ is the product of its maximal localisations. We thus have a splitting $R=\prod_{i=1}^{n} R_{i}$ say, where each factor $R_{i}$ an Artinian local ring. It follows that the lattice of ideals in $R$ is the product of the corresponding lattices for the factors $R_{i}$, and thus that each $R_{i}$ satisfies condition (b). We can thus reduce to the case where $R$ is local, with maximal ideal $\mathfrak{m}$ say. Recall that the socle is $\operatorname{soc}(R)=\{a \in R \mid a \mathfrak{m}=0\}=\operatorname{ann}_{R}(\mathfrak{m})$, which is naturally a vector space over the field $K=R / \mathfrak{m}$. If $\operatorname{soc}(R)$ were zero we would have $\mathfrak{m}=\operatorname{ann}^{2}(\mathfrak{m})=\operatorname{ann}(\operatorname{soc}(R))=\operatorname{ann}(0)=R$, which is a contradiction. We can therefore choose a nonzero element $u \in \operatorname{soc}(R)$. We find that $K u=R u$ is a nonzero ideal in $R$, so $\operatorname{ann}(K u)$ is a proper ideal containing $\operatorname{ann}(\operatorname{soc}(R))=\mathfrak{m}$, so $\operatorname{ann}(K u)=\mathfrak{m}$ by maximality. We can now take annihilators again to see that $K u=\operatorname{ann}(\mathfrak{m})=\operatorname{soc}(R)$, so $\operatorname{soc}(R)$ is one-dimensional. This proves (c).

Finally, we will assume (c) and prove (a). It is again easy to reduce to the case where $R$ is local, and the local case is covered by Corollary 4.4 .

Definition 4.5. Let $K$ be a field. A Poincaré duality algebra over $K$ is a graded commutative $K$-algebra $R$ equipped with a $K$-linear map $\theta: R_{d} \rightarrow K$ for some $d \geq 0$ such that:

- For $i<0$ or $i>d$ we have $R_{i}=0$.

- $R_{0}=K$.

- For $0 \leq i \leq d$ we have $\operatorname{dim}_{K}\left(R_{i}\right)<\infty$, and the map $(a, b) \mapsto \theta(a b)$ defines a perfect pairing between $R_{i}$ and $R_{d-i}$.

Proposition 4.6. Every Poincaré duality algebra is self-injective.

Proof. Let $R$ be a Poincaré duality algebra of top dimension $d$, and put $\mathfrak{m}=\bigoplus_{i>0} R_{i}$. It is clear that $R / \mathfrak{m}=K$ and $\mathfrak{m}^{d+1}=0$, and it follows that $\mathfrak{m}$ is the unique maximal 
ideal. As $R$ has finite total dimension over $K$ it is clearly Artinian. The perfect pairing condition implies that $\operatorname{soc}(R)=R_{d}$ and that this has dimension one. It follows by Theorem 4.1 that $R$ is self-injective.

Alternatively, for any $R$-module $M$ we can define a natural map

$$
\tau: \operatorname{Hom}_{R}(M, R) \rightarrow \operatorname{Hom}_{K}\left(M_{d}, K\right)
$$

by $\tau(\phi)=\theta \circ \phi_{d}$. Using the perfectness of the pairing we see that this is an isomorphism. As $K$ is a field, the functor $M \mapsto \operatorname{Hom}_{K}\left(M_{d}, K\right)$ is exact, and it follows that the functor $M \mapsto \operatorname{Hom}_{R}(R, R)$ is also exact, or in other words that $R$ is injective as an $R$-module.

Example 4.7. Put

$$
E=\mathbb{F}\left[x_{0}, x_{1}, x_{2}, \ldots\right] /\left(x_{i}^{2} \mid i \geq 0\right),
$$

with $\left|x_{i}\right|=2^{i}$. For any finite set $I \subset \mathbb{N}$ we put $x_{I}=\prod_{i \in I} x_{i}$, so $\left|x_{I}\right|=\sum_{i \in I} 2^{i}$ and the elements $x_{I}$ form a basis for $E$ over $\mathbb{F}$. It follows that $E_{k} \simeq \mathbb{E}$ for all $k \geq 0$, and $E_{k}=0$ for $k<0$. Let $E(n)$ be the subalgebra of $E$ generated by $x_{0}, \ldots, x_{n-1}$. This is a Poincaré duality algebra, with socle generated by the element $\prod_{i<n} x_{i}$, and it is clear that $E=\bigcup_{n} E(n)$. Corollary 3.7 therefore tells us that $E$ is self-injective.

\section{Coherence}

We now briefly recall some standard ideas about finite presentation.

Definition 5.1. Let $R$ be a graded commutative ring, and let $M$ be a graded $R$-module. Then we see from [Lam 1999, Section 4D] the following are equivalent:

(a) There exists an exact sequence

$$
P_{1} \stackrel{f}{\rightarrow} P_{0} \stackrel{g}{\rightarrow} M \rightarrow 0
$$

where $P_{0}$ and $P_{1}$ are finitely generated free modules.

(b) $M$ is finitely generated, and for every epimorphism $g: P_{0} \rightarrow M$ (with $P_{0}$ a finitely generated free module) the module $\operatorname{ker}(g)$ is also finitely generated.

If these conditions hold, we say that $M$ is finitely presented.

Remark 5.2. By finitely generated free module, we mean one of the form $\bigoplus_{i=1}^{r} \Sigma^{d_{i}} R$; we do not assume that the degree shift $d_{i}$ is zero.

Corollary 5.3. If $R$ is Noetherian, then every finitely generated ideal is finitely presented.

Proof. Condition (b) is clearly satisfied. 
As we stated in Definition 1.2, a graded ring $R$ is said to be coherent if every finitely generated ideal is finitely presented, and totally incoherent if the only finitely presented ideals are 0 and $R$. It is clear that every Noetherian ring is coherent. We mention as background that if $R$ is coherent, then the category of finitely generated modules is closed under images, kernels, cokernels and extensions, so it is an abelian category. The following example is standard:

Proposition 5.4. The infinite exterior algebra E (as in Example 4.7) is coherent.

Proof. Let $E(n)$ be the subalgebra generated by $x_{0}, \ldots, x_{n-1}$, and let $E^{\prime}(n)$ be generated by the remaining variables, so $E=E(n) \otimes_{\mathbb{F}} E^{\prime}(n)$. Any finitely generated ideal is the image of some $E$-linear map $g: E^{r} \rightarrow E$, which will have the form $g(u)=u . v$ for some vector $v \in E^{r}$. We must show that the module $K=\operatorname{ker}(g)$ is finitely generated. Choose $n$ large enough that $v_{i} \in E(n)$ for all $i$. Now $v$ gives a map $g^{\prime}: E(n)^{r} \rightarrow E(n)$ of $E(n)$-modules, and $E(n)$ is Noetherian, so the module $K^{\prime}=\operatorname{ker}\left(g^{\prime}\right)$ is finitely generated over $E(n)$. We can identify $g$ with $g^{\prime} \otimes 1$ with respect to the splitting $E=E(n) \otimes E^{\prime}(n)$, and it follows that $K=K^{\prime} \otimes E(n)^{\prime}$, and thus that any finite generating set for $K^{\prime}$ over $E(n)$ also generates $K$ over $E$.

The following result will be our main tool for proving incoherence results.

Lemma 5.5. Let $A$ be a local graded ring, with maximal ideal $\mathfrak{m}$, and let $I$ be a finitely presented ideal in $A$. Then for each $u \in I \backslash \mathfrak{m} I$, the image of $\operatorname{ann}_{A}(u)$ in $\mathfrak{m} / \mathfrak{m}^{2}$ has finite dimension over $A / \mathfrak{m}$.

Note here that as $u \notin \mathfrak{m} I$ we have $u \neq 0$, so $\operatorname{ann}_{A}(u) \leq \mathfrak{m}$ and it is meaningful to talk about the image in $\mathfrak{m} / \mathfrak{m}^{2}$.

Proof. As $I$ is finitely generated over $A$, we see that $I / \mathfrak{m} I$ is a finite-dimensional vector space over $A / \mathfrak{m}$. We can choose a basis for this space containing the image of $u$, and then choose elements of $I$ lifting these basis elements. This gives a list $v_{1}, \ldots, v_{n} \in I$ with $v_{1}=u$ such that the corresponding map $g: A^{n} \rightarrow I$ induces an isomorphism $\bar{g}:(A / \mathfrak{m})^{n} \rightarrow I / \mathfrak{m} I$. Now $\operatorname{cok}(g)$ is a finitely generated module with $\mathfrak{m} \cdot \operatorname{cok}(g)=\operatorname{cok}(g)$, so $\operatorname{cok}(g)=0$ by Nakayama's lemma, and so $g$ is an epimorphism. As $I$ is assumed to be finitely presented, we see that $\operatorname{ker}(g)$ is also finitely generated over $A$. Moreover, as $\bar{g}$ is an isomorphism we see that $\operatorname{ker}(g) \leq \mathfrak{m}^{n}$. It follows that the image of $\operatorname{ker}(g)$ in $\left(\mathfrak{m} / \mathfrak{m}^{2}\right)^{n}$ is finite-dimensional. The intersection of $\operatorname{ker}(g)$ with the first copy of $A$ in $A^{n}$ is just the annihilator of $u$, so we see that the image of $\operatorname{ann}_{A}(u)$ in $\mathfrak{m} / \mathfrak{m}^{2}$ is finite-dimensional.

Corollary 5.6. Let A be a local graded ring, with maximal ideal $\mathfrak{m}$. Suppose that all $u \in A$ satisfy one of the following conditions: $u=0$; the image of $\operatorname{ann}_{A}(u)$ in $\mathfrak{m} / \mathfrak{m}^{2}$ has infinite dimension; or $u$ is invertible.

Then A is totally incoherent. 
Proof. Let $I$ be a finitely presented ideal. If $\mathfrak{m} I=I$ then $I=0$ by Nakayama's lemma. Otherwise, we can choose $u \in I \backslash \mathfrak{m} I$. As $u \notin \mathfrak{m} I$ we have $u \neq 0$. By the lemma, the image of $\operatorname{ann}_{A}(u)$ in $\mathfrak{m} / \mathfrak{m}^{2}$ must have finite dimension. Thus, the first two possibilities are excluded, and $u$ must be invertible. As $u \in I$ we conclude that $I=A$.

Next we record a graded version of Chase's theorem for coherent rings.

Theorem 5.7. Let $R$ be a graded commutative ring. The following conditions are equivalent:

(a) $R$ is coherent.

(b) For all elements $a \in R$ and for every finitely generated ideal $J \leq R$, the conductor ideal

$$
(J: a)=\{r \in R \mid r a \in J\}
$$

is finitely generated.

(c) For all elements $a \in R$, the annihilator ideal $\operatorname{ann}_{R}(a)$ is finitely generated, and for all finitely generated ideals $J, K \leq R$, the intersection $J \cap K$ is finitely generated.

Proof. The ungraded version of the proof is given in many textbooks such as [Lam 1999, page 142]. It can be modified in an obvious way to keep track of gradings, which gives our statement above.

Theorem 5.8. Let $R$ be a graded commutative ring such that $R_{k}$ is finite for all $k$. The following conditions are equivalent:

(a) $R$ is coherent and self-injective.

(b) $R$ is coherent and for all finitely generated ideals $J \leq R$ we have $\operatorname{ann}_{R}^{2}(J)=J$.

(c) For every finitely generated ideal $J \leq R$, the ideal $\operatorname{ann}_{R}(J)$ is finitely generated and $\operatorname{ann}_{R}^{2}(J)=J$.

(d) $R$ is self injective and for all finitely generated ideals $J \leq R$, the ideal $\operatorname{ann}_{R}(J)$ is finitely generated.

Proof. It follows from Remark 2.4 that (a) implies (b). To show that (b) implies (c) we need to show that the ideal $\operatorname{ann}_{R}(J)$ is finitely generated for each finitely generated ideal $J \leq R$. If we let $\left(r_{1}, \ldots, r_{n}\right)$ be generators for the ideal $J$, then we can take the annihilator of $J$ to give $\operatorname{ann}_{R}(J)=\bigcap_{i} \operatorname{ann}_{R}\left(r_{i}\right)$. Since $R$ is assumed to be coherent, it follows from part (c) of Theorem 5.7 that $\operatorname{ann}_{R}\left(r_{i}\right)$ is finitely generated for each $i$ and that a finite intersection of finitely generated ideals is also finitely generated. Thus $\operatorname{ann}_{R}(J)$ is finitely generated as claimed. Now suppose 
that part (c) holds. To prove that (c) implies (d), we need to show that $R$ is injective as an $R$-module. For all ideals $J, K \leq R$ we have

$$
\operatorname{ann}_{R}\left(\operatorname{ann}_{R}(J)+\operatorname{ann}_{R}(K)\right)=\operatorname{ann}_{R}^{2}(J) \cap \operatorname{ann}_{R}^{2}(K)=J \cap K .
$$

By assumption, the ideal sum $\operatorname{ann}_{R}(J)+\operatorname{ann}_{R}(K)$ must be finitely generated. Thus we can take double annihilators to give

$$
\operatorname{ann}_{R}(J)+\operatorname{ann}_{R}(K)=\operatorname{ann}_{R}(J \cap K) .
$$

Since $R_{k}$ is finite for each $k$, we can use part (b) of Theorem 3.8 to complete the claim. We now conclude by showing that (d) implies (a). By assumption, the annihilator ideal $\operatorname{ann}_{R}(a)$ is finitely generated for all elements $a \in R$. Then for all ideals $J, K \leq R$ we know that the ideal sum $\operatorname{ann}_{R}(J)+\operatorname{ann}_{R}(K)$ is finitely generated by assumption. By taking annihilators we then have

$$
\operatorname{ann}_{R}\left(\operatorname{ann}_{R}(J)+\operatorname{ann}_{R}(K)\right)=\operatorname{ann}_{R}^{2}(J) \cap \operatorname{ann}_{R}^{2}(K)=J \cap K,
$$

where the double annihilator condition holds by Remark 2.4. However, by assumption, the annihilator of a finitely generated ideal is also finitely generated. Thus the intersection $J \cap K$ must be finitely generated. It follows from part (c) of Theorem 5.7 that the ring $R$ is coherent as claimed.

\section{Self-injective adjustment}

Definition 6.1. We write $\mathscr{R}$ for the category of commutative graded $\mathbb{F}$-algebras such that:

(a) $R_{k}=0$ for all $k<0$.

(b) $R_{0}=\mathbb{F}$.

(c) $R_{k}$ is finite for all $k>0$.

Proposition 6.2. Let $R$ be a ring in $\mathscr{R}$, and let $\mathscr{P}$ be a finite set of test pairs in $R$ that have no transporters. Let $m$ be a positive integer. Then there is an extension $R^{\prime} \geq R$ of graded rings such that:

(a) $R^{\prime}$ is also in $\mathscr{R}$.

(b) $R_{k}^{\prime}=R_{k}$ for all $k<m$.

(c) Each test pair in $\mathscr{P}$ has a block in $R^{\prime}$.

Proof. List the elements of $\mathscr{P}$ as $\left(u_{0}, v_{0}\right), \ldots,\left(u_{p-1}, v_{p-1}\right)$ say. Suppose that $\left(u_{t}, v_{t}\right)$ has length $r_{t}$, and let $d_{t}$ be the maximum of the degrees of the entries $u_{t, j}$ for $0 \leq j<r_{t}$. Let $P$ be the polynomial ring obtained from $R$ by adjoining variables $b_{t, j}$ for $0 \leq t<p$ and $0 \leq j<r_{t}$, with $\left|b_{t, j}\right|=m+d_{t}-\left|u_{t, j}\right| \geq m>0$. Put $w_{t}=\sum_{j=0}^{r_{t}-1} b_{t, j} u_{t, j} \in P$ and $R^{\prime}=P /\left(w_{0}, \ldots, w_{p-1}\right)$. There is an evident ring map 
$\eta: R \rightarrow R^{\prime}$, and also a ring map $\pi: R^{\prime} \rightarrow R$ given by $\pi\left(b_{t, j}\right)=0$ for all $t$ and $j$. It is clear that $\pi \eta=1$, so $\eta$ is injective, and we can use it to regard $R^{\prime}$ as an extension of $R$. As $\left|b_{t, j}\right| \geq m>0$, it is easy to see that $R^{\prime} \in \mathscr{R}$ and that the map $R_{k} \rightarrow R_{k}^{\prime}$ is surjective (and therefore bijective) for $k<m$. By construction we have $b_{t} \cdot u_{t}=0$ in $R^{\prime}$. We claim that $b_{t} . v_{t} \neq 0$ in $R^{\prime}$, or equivalently that $b_{t} \cdot v_{t}$ cannot be written as $\sum_{s} c_{s} w_{s}$ in $P$. To see this, let $c^{*}$ denote the constant term in the polynomial $c_{t}$. By examining the coefficient of $b_{t, j}$ in the equation $b_{t} \cdot v_{t}=\sum_{s} c_{s} w_{s}$ we obtain $v_{t, j}=c^{*} u_{t, j}$ for all $j$, which means that $c^{*}$ is a transporter for $\left(u_{t}, v_{t}\right)$, contrary to assumption. Thus, $b_{t}$ is a block for $\left(u_{t}, v_{t}\right)$ in $R^{\prime}$, as required.

Definition 6.3. Let $R$ be a ring in $\mathscr{R}$, and let $(u, v)$ be a test pair for $R$. We say that $(u, v)$ is good if it has either a block or a transporter, and bad otherwise. We say that $(u, v)$ is nondegenerate if $u_{i} \neq 0$ for all $i$. For any homogeneous element $x \in R$ we put $|x|_{+}=\max (0,|x|)$. The weight of $(u, v)$ is $\sum_{i}\left(1+\left|u_{i}\right|_{+}+\left|v_{i}\right|_{+}\right)$.

Lemma 6.4. Let $R$ be a ring in $\mathscr{R}$, and suppose that all nondegenerate test pairs are good. Then $R$ is self-injective.

Proof. Consider an arbitrary test pair $(u, v) \in R^{r} \times R^{r}$. If there exists $i$ such that $u_{i}=0$ but $v_{i} \neq 0$, then the basis vector $e_{i} \in R^{r}$ is a block for $(u, v)$. Otherwise, let $\left(u^{\prime}, v^{\prime}\right)$ be the test pair obtained by removing all zeros from $u$ and the corresponding zeros from $v$. This is nondegenerate, so it has a block or a transporter. If $b^{\prime}$ is a block for $\left(u^{\prime}, v^{\prime}\right)$, then we can construct a block for $(u, v)$ by inserting some zeros. If $m^{\prime}$ is a transporter for $\left(u^{\prime}, v^{\prime}\right)$, then it is also a transporter for $(u, v)$. We therefore see that all test pairs for $R$ are good, so $R$ is self-injective.

Lemma 6.5. There are only finitely many nondegenerate bad test pairs of any given weight.

Proof. Consider an integer $N \geq 0$. Any nondegenerate bad test pair $(u, v)$ of weight $N$ must have length at most $N$. Moreover, as $(u, v)$ is nondegenerate we must have $u_{i} \neq 0$ for all $i$, and as $R \in \mathscr{R}$ this means that $\left|u_{i}\right| \geq 0$. We also have $\sum_{i}\left|u_{i}\right| \leq$ weight $(u, v)=N$. It is clear from this (and the finiteness of $R_{k}$ ) that there are only finitely many possibilities for $u$. Next, let $d$ be the degree of $(u, v)$, so $\left|v_{i}\right|=\left|u_{i}\right|+d$. From this it is clear that $d \leq N$. If $d$ is sufficiently negative then we will have $v_{i}=0$ for all $i$, so 0 is a transporter for $(u, v)$, contradicting the assumption that $(u, v)$ is bad. We therefore see that there are only finitely many possibilities for $d$. Given $u$ and $d$, it is clear that there are only finitely many possibilities for $v$.

Theorem 6.6. Suppose that $R \in \mathscr{R}$, and that $m \geq 0$. Then there is an extension $R^{\prime} \geq R$ such that:

(a) $R^{\prime}$ is also in $R$.

(b) $R_{k}^{\prime}=R_{k}$ for all $k<m$. 
(c) $R^{\prime}$ is self-injective.

Proof. We define rings $R^{\prime}(0) \leq R^{\prime}(1) \leq \cdots$ as follows. We start with $R^{\prime}(0)=R$. For each $k \geq 0$, we let $R^{\prime}(k+1)$ be an extension of $R^{\prime}(k)$ that agrees with $R^{\prime}(k)$ in degrees less than $k+m$, such that every nondegenerate bad test pair of weight at most $k$ in $R^{\prime}(k)$ has a block in $R^{\prime}(k+1)$. This can be constructed by Proposition 6.2 and Lemma 6.5. Now take $R^{\prime}$ to be the colimit of the rings $R^{\prime}(k)$. By construction we have $R_{i}^{\prime}=R^{\prime}(k)_{i}$ for sufficiently large $k$, and using this it is clear that $R^{\prime} \in \mathscr{R}$. Consider a nondegenerate test pair $(u, v) \in R^{\prime}$. For sufficiently large $k$ we can assume that $k \geq \operatorname{weight}(u, v)$ and that $u_{i}, v_{i} \in R^{\prime}(k)$ for all $i$. If $(u, v)$ is good in $R^{\prime}(k)$ then it is good in $R^{\prime}$. If it is bad in $R^{\prime}(k)$ then by construction it becomes good in $R^{\prime}(k+1)$ and therefore in $R^{\prime}$.

\section{The cube algebra}

Recall that in the statement of Theorem 1.6 we introduced the ring

$$
C=\mathbb{F}\left[y_{0}, y_{1}, \ldots\right] /\left(y_{i}^{3}+y_{i} y_{i+1} \mid i \geq 0\right),
$$

with the grading given by $\left|y_{i}\right|=2^{i}$. We now investigate the structure of this ring (which we call the cube algebra).

Definition 7.1. We also put

$$
\begin{aligned}
C[n, \infty] & =\mathbb{F}\left[y_{n}, y_{n+1}, \ldots\right] /\left(y_{i}^{3}+y_{i} y_{i+1} \mid n \leq i<\infty\right), \\
C[n, m] & =\mathbb{F}\left[y_{n}, \ldots, y_{m}\right] /\left(y_{i}^{3}+y_{i} y_{i+1} \mid n \leq i<m\right), \\
\bar{C}[n, m] & =C[n, m] / y_{m} .
\end{aligned}
$$

Lemma 7.2. The evident maps

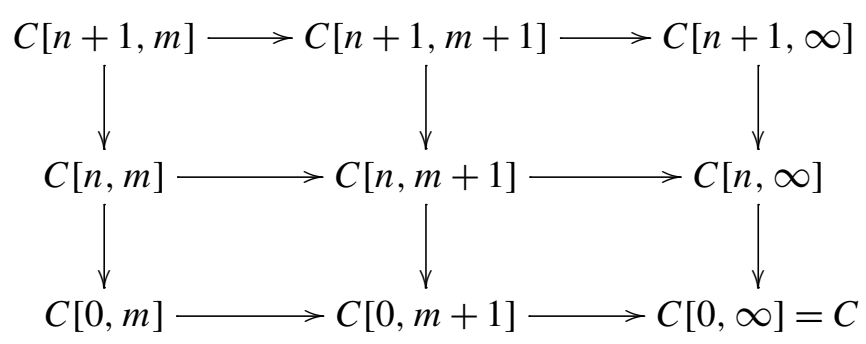

are all split injective, so all the rings mentioned can be considered as subrings of $C$. Proof. There is a graded ring map $\tau_{0}: \mathbb{F}\left[y_{0}, y_{1}, \ldots\right] \rightarrow C[n, m]$ given by

$$
\tau_{0}\left(y_{i}\right)= \begin{cases}0 & \text { if } i<n, \\ y_{i} & \text { if } n \leq i \leq m, \\ y_{m}^{2^{i-m}} & \text { if } m \leq i\end{cases}
$$


It is straightforward to check that $\tau_{0}\left(y_{i}^{3}+y_{i} y_{i+1}\right)=0$ for all $i \geq 0$, so there is an induced map $\tau: C \rightarrow C[n, m]$. Clearly the composite $C[n, m] \rightarrow C \stackrel{\tau}{\rightarrow} C[n, m]$ is the identity, so the map $C[n, m] \rightarrow C$ is injective for all $m$ and $n$. The other claims follow from this.

Definition 7.3. We write $P$ for the polynomial ring $\mathbb{F}\left[y_{0}, y_{1}, \ldots\right]$, so that $C$ is a quotient of $P$. A multiindex is a sequence $\alpha=\left(\alpha_{0}, \alpha_{1}, \ldots\right)$ of natural numbers with $\alpha_{i}=0$ for $i \gg 0$. We write $M P$ for the set of all multiindices. Given $\alpha \in M P$ we write $y^{\alpha}=\prod_{i} y_{i}^{\alpha_{i}}$ and $|\alpha|=\left|y^{\alpha}\right|=\sum_{i} \alpha_{i} 2^{i}$. It is clear that the set $B P=\left\{y^{\alpha} \mid \alpha \in M P\right\}$ is a basis for $P$ over $\mathbb{F}$.

Definition 7.4. We put

$$
\begin{aligned}
M^{\prime} C[n, m] & =\left\{\alpha \in M P \mid \alpha_{i}=0 \text { for } i<n \text { or } i>m \text { and } \alpha_{i}<3 \text { for } n \leq i<m\right\}, \\
M \bar{C}[n, m] & =\left\{\alpha \in M P \mid \alpha_{i}=0 \text { for } i<n \text { or } i \geq m\right\}, \\
B^{\prime} C[n, m] & =\left\{y^{\alpha} \mid \alpha \in M^{\prime} C[n, m]\right\}, \\
B \bar{C}[n, m] & =\left\{y^{\alpha} \mid \alpha \in M \bar{C}[n, m]\right\} .
\end{aligned}
$$

Note that in the definition of $M^{\prime} C[n, m]$ the constraint $\alpha_{i}<3$ does not apply when $i=m$, so in particular $M^{\prime} C[n, m]$ is infinite.

Proposition 7.5. $B^{\prime} C[n, m]$ is a basis for $C[n, m]$, and $B \bar{C}[n, m]$ is a basis for $\bar{C}[n, m]$. Moreover, $\bar{C}[n, m]$ is a Poincaré duality algebra over $\mathbb{F}$.

The proof depends on the following result:

Lemma 7.6. Let $A$ be a commutative algebra over $\mathbb{F}$, let $f(t) \in A[t]$ be a monic polynomial of degree $d$, and put $B=A[x] / f(x)$. Then $\left\{1, x, \ldots, x^{d-1}\right\}$ is a basis for $B$ over A. Moreover, if $A$ is finite-dimensional over $\mathbb{F}$ and has Poincaré duality, then the same is true of $B$.

Proof. We first claim that any polynomial $g(x) \in A[x]$ can be expressed uniquely in the form $g(x)=q(x) f(x)+r(x)$ with $\operatorname{deg}(r(x))<d$. This can easily be proved by induction on the degree of $g(x)$, and it follows directly that $\left\{1, \ldots, x^{d-1}\right\}$ is a basis for $B$ over $A$. Now suppose that $A$ has Poincaré duality, so there is a linear map $\theta: A \rightarrow \mathbb{F}$ such that the bilinear form $(u, v) \mapsto \theta(u, v)$ is perfect. This means that there exist bases $\left\{u_{0}, \ldots, u_{n-1}\right\}$ and $\left\{v_{0}, \ldots, v_{n-1}\right\}$ for $A$ such that $\theta\left(u_{i} v_{j}\right)=\delta_{i j}$. Now define $\phi: B \rightarrow \mathbb{F}$ by $\phi\left(\sum_{i=0}^{d-1} a_{i} x^{i}\right)=\theta\left(a_{d-1}\right)$. We define bases $\left\{s_{0}, \ldots, s_{n d-1}\right\}$ and $\left\{t_{0}, \ldots, t_{n d-1}\right\}$ for $B$ by $s_{n i+j}=x^{i} u_{j}$ and $t_{n i+j}=x^{d-1-i} v_{j}$ for $0 \leq i<d$ and $0 \leq j<n$. It is clear that $\phi\left(s_{k} t_{k}\right)=1$. Suppose we have $0 \leq k<k^{\prime}<n d$. Write $k=n i+j$ and $k^{\prime}=n i^{\prime}+j^{\prime}$ as before; we must have either $i<i^{\prime}$, or $\left(i=i^{\prime}\right.$ and $\left.j<j^{\prime}\right)$. In either case, we find that $\phi\left(s_{i} t_{j}\right)=0$. Thus, the matrix of $\phi$ with respect to our bases is triangular, with ones on the diagonal, proving that $\phi$ gives a perfect pairing on $B$. 
Proof of Proposition 7.5. From the definitions we have $C[m, m]=\mathbb{F}\left[y_{n}\right]$ and $B^{\prime} C[m, m]=\left\{y_{n}^{\alpha_{n}} \mid \alpha_{n} \in \mathbb{N}\right\}$ so clearly $B^{\prime} C[m, m]$ is a basis for $C[m, m]$. Similarly, it is clear that the set $\bar{C}[m, m]=\{1\}$ is a basis for the ring $\bar{C}[m, m]=C[m, m] / y_{m}=\mathbb{F}$, and that this has Poincaré duality.

Next, $C[n, m]$ can be described as

$$
C[n+1, m]\left[y_{n}\right] / f\left(y_{n}\right),
$$

where $f(t)=t^{3}+y_{n+1} t$ is a monic polynomial of degree three with coefficients in $C[n+1, m]$. It also follows that $\bar{C}[n, m]=\bar{C}[n+1, m]\left[y_{n}\right] / f\left(y_{n}\right)$. All claims in the proposition now follow by downwards induction on $n$ using Lemma 7.6.

Remark 7.7. Note that the algebra

$$
\bar{C}[n, m]=\frac{\mathbb{F}\left[y_{n}, y_{n+1}, \ldots, y_{m-1}\right]}{\left(y_{n}^{3}+y_{n} y_{n+1}, \ldots, y_{m-1}^{3}\right)}
$$

has the same number of relations as generators, and has finite dimension over $\mathbb{F}$. It is known that in this situation the sequence of relations is necessarily regular, and that the algebra automatically has Poincare duality. (This can be extracted from [Matsumura 1980, Section 17], for example.) This would give another approach to Proposition 7.5.

Definition 7.8. Let $\alpha$ be a multiindex. We say that

(a) $\alpha$ is flat if $\alpha_{i}<3$ for all $i$;

(b) $\alpha$ is $n$-truncated if $\alpha_{i}=0$ for all $i<n$;

(c) $\alpha$ is $m$-solid if it is flat and whenever $m \leq p \leq q$ and $\alpha_{q}>0$ we also have $\alpha_{p}>0$.

We consider all flat multiindices to be $\infty$-solid. For $0 \leq n \leq m \leq \infty$ we put

$$
M C[n, m]=\{\alpha \in M P \mid \alpha \text { is } n \text {-truncated and } m \text {-solid }\},
$$

and $B C[n, m]=\left\{y^{\alpha} \mid \alpha \in M C[n, m]\right\}$. We also write $M C$ for the set $M C[0, \infty]$ of all flat multiindices.

Proposition 7.9. $B C[n, \infty]$ is a basis for $C[n, \infty]$.

Proof. We must show that for each degree $d \in \mathbb{N}$, the set $B C[n, \infty]_{d}$ is a basis for $C[n, \infty]_{d}$. Choose $m>n$ such that $2^{m}>d$. Then clearly $B C[n, \infty]_{d}=B^{\prime} C[n, m]_{d}$ and $C[n, \infty]_{d}=C[n, m]_{d}$ so the claim follows from Proposition 7.5.

It is also true that $B C[n, m]$ is a basis for $C[n, m]$ when $m<\infty$, but it is convenient to leave the proof until later.

Proposition 7.10. For any multiindex $\alpha \in M P$, there is a multiindex $\beta \in M C$ such that $y^{\alpha}=y^{\beta}$. 
Proof. If $\alpha \notin M C$, we let $k$ denote the smallest index such that $\alpha_{k}>2$, and define $\alpha^{\prime} \in M P$ by

$$
\alpha_{i}^{\prime}= \begin{cases}\alpha_{i} & \text { if } i<k \\ \alpha_{k}-2 & \text { if } i=k, \\ \alpha_{k+1}+1 & \text { if } i=k+1 \\ \alpha_{i} & \text { if } i>k+1\end{cases}
$$

Because $y_{k}^{3}=y_{k} y_{k+1}$ we have $y^{\alpha}=y^{\alpha^{\prime}}$. Moreover, $\alpha^{\prime}$ has the same degree as $\alpha$, and is lexicographically lower than $\alpha$. There are only finitely many monomials of any given degree, so the claim follows by induction over the lexicographic order.

Definition 7.11. (a) We put $x_{0}=y_{0}$, and $x_{n}=y_{n}+y_{n-1}^{2}$ for all $n>0$.

(b) For $n \geq m \geq 0$ we put $x_{[m, n]}=\prod_{i=n}^{m} x_{i}$ and $y_{[m, n]}=\prod_{i=n}^{m} y_{i}$.

Proposition 7.12. For all $n \geq 0$ we have $y_{n}=\sum_{i=0}^{n} x_{n-i}^{2^{i}}$ and $y_{n} x_{n+1}=0$. Thus, the ring $C$ can also be presented as

$$
C=\mathbb{F}\left[x_{0}, x_{1}, x_{2}, \ldots\right] /\left(x_{n+1} \sum_{i=0}^{n} x_{n-i}^{2^{i}} \mid n \geq 0\right) .
$$

Proof. Once we recall that $(a+b)^{2}=a^{2}+b^{2}(\bmod 2)$, the equation $y_{n}=\sum_{i=0}^{n} x_{n-i}^{2^{i}}$ is easily checked by induction. Note that this already holds in the polynomial ring $P$. As the elements $x_{i}$ can be expressed in terms of the $y_{j}$ and vice versa, we see that $P=\mathbb{F}\left[x_{0}, x_{1}, \ldots\right]$. The defining relations $y_{n}^{3}+y_{n} y_{n+1}=0$ for $C$ can clearly be rewritten as $y_{n} x_{n+1}=0$ and thus as $x_{n+1} \sum_{i=0}^{n} x_{n-i}^{2^{i}}=0$.

Lemma 7.13. Whenever $m \leq n$ we have $y_{m} y_{[m, n]}^{2}=y_{[m, n+1]}$.

Proof. The inductive step is

$$
\begin{aligned}
y_{m} y_{[m, n+1]}^{2} & =y_{m} y_{[m, n]}^{2} y_{n+1}^{2}=y_{[m, n+1]} y_{n+1}^{2} \\
& =y_{[m, n]} y_{n+1}^{3}=y_{[m, n]} y_{n+1} y_{n+2}=y_{[m, n+2]} .
\end{aligned}
$$

Corollary 7.14. For $k \geq 0$ we have $y_{m}^{2^{k}-1}=y_{[m, m+k-1]}$.

Proof. The induction step is

$$
y_{m}^{2^{k+1}-1}=y_{m}\left(y_{m}^{2^{k}-1}\right)^{2}=y_{m} y_{[m, m+k-1]}^{2}=y_{[m, m+k]} .
$$

Lemma 7.15. Fix $m \in \mathbb{N}$, and put

$$
U=\left\{\alpha \in M C \mid \alpha \text { is } m \text {-solid and } \alpha_{i}=0 \text { for } i<m\right\} .
$$

Then there is a bijection $\mathbb{N} \rightarrow U$ written as $k \mapsto \theta[m, k]$ such that $y^{\theta[m, k]}=y_{m}^{k}$ in $C$. 
Proof. First, if $\alpha \in U$ it is clear that $|\alpha|$ is divisible by $2^{m}$, so we can define $\delta: U \rightarrow \mathbb{N}$ by $\delta(\alpha)=|\alpha| / 2^{m}$.

Now consider $k \in \mathbb{N}$. There is a unique $r \in \mathbb{N}$ such that $2^{r}-1 \leq k<2^{r+1}-1$. This means that $0 \leq k-\left(2^{r}-1\right)<2^{r}$, so there is a unique set $J \subseteq\{0,1, \ldots, r-1\}$ with $k-\left(2^{r}-1\right)=\sum_{j \in J} 2^{j}$. We put

$$
\theta[m, k]_{i}= \begin{cases}0 & \text { if } i<m, \\ 1 & \text { if } m \leq i<m+r \text { and } i-m \notin J, \\ 2 & \text { if } m \leq i<m+r \text { and } i-m \in J, \\ 0 & \text { if } m+r \leq i .\end{cases}
$$

This is clearly in $U$. Next, we claim that $y^{\theta[m, k]}=y_{m}^{k}$. To see this, put $z=y_{m}^{2^{r}-1}$, which is the same as $y_{[m, m+r-1]}$ by Corollary 7.14. We have

$$
\begin{gathered}
y^{\theta[m, k]}=y_{[m, m+r-1]} \prod_{j \in J} y_{m+j}=z \prod_{j \in J} y_{m+j}, \\
y_{m}^{k}=y_{m}^{2^{r}-1+\sum_{j \in J} 2^{j}}=z \prod_{j \in J} y_{m}^{2^{j}} .
\end{gathered}
$$

Now, for $0 \leq j<r$ we have $y_{m+j}\left(y_{m+j}^{2}+y_{m+j+1}\right)=0$ and $z$ is divisible by $y_{m+j}$ so $z\left(y_{m+j}^{2}+y_{m+j+1}\right)=0$, and so $y_{m+j+1}=y_{m+j}^{2}$ modulo ann $(z)$. It follows inductively that $y_{m+j}=y_{m}^{2^{j}}(\bmod \operatorname{ann}(z))$, so $\prod_{j \in J} y_{m+j}=\prod_{j \in J} y_{m}^{2^{j}}(\bmod \operatorname{ann}(z))$, so $y^{\theta[m, k]}=y_{m}^{k}$ as claimed. It also follows that $\delta(\theta[m, k])=\left|y^{\theta[m, k]}\right| / 2^{m}=\left|y_{m}^{k}\right| / 2^{m}=k$.

Now let $\alpha$ be an arbitrary element of $U$. By the definition of solidity, there is an integer $s \geq 0$ such that when $m \leq i<m+s$ we have $\alpha_{i} \in\{1,2\}$ and for $i \geq m+s$ we have $\alpha_{i}=0$. It is then clear that

$$
\sum_{m \leq i<m+s} 2^{i} \leq|\alpha| \leq 2 \sum_{m \leq i<m+s} 2^{i}
$$

or in other words $2^{s}-1 \leq \delta(\alpha)<2^{s+1}-1$. It follows easily that $\alpha=\theta[m, \delta(\alpha)]$, so we have a bijection as claimed.

Proposition 7.16. For $0 \leq n \leq m \leq \infty$, the set $B C[n, m]$ is a basis for $C[n, m]$.

Proof. The case $m=\infty$ was covered by Proposition 7.9, so we may assume that $m<\infty$, so $B^{\prime} C[n, m]$ is a basis for $C[n, m]$ by Proposition 7.5. However, Lemma 7.15 implies that $B^{\prime} C[n, m]$, considered as a system of elements in $C[n, m]$, is just the same as $B C[n, m]$.

Proposition 7.17. Suppose that $0 \leq n<k \leq m \leq \infty$ and $k<\infty$. Then

$$
\operatorname{ann}_{C[n, m]}\left(x_{k}\right)=C[n, m] y_{k-1} .
$$


Proof. The $m=\infty$ case will follow from the $m<\infty$ case, as $C[n, m]_{d}=C[n, \infty]_{d}$ when $m$ is large relative to $d$. We will thus assume that $m<\infty$.

We have already observed that $x_{k} y_{k-1}=0$, so $\operatorname{ann}_{C[n, m]}\left(x_{k}\right) \geq C[n, m] y_{k-1}$, and multiplying by $x_{k}$ gives a well-defined map $f: C[n, m] /\left(C[n, m] y_{k-1}\right) \rightarrow C[n, m]$. It will suffice to show that $f$ is injective.

For this, we put

$$
\begin{aligned}
& N=\left\{\alpha \in M C[n, m] \mid \alpha_{k-1}=0\right\}, \\
& A=\left\{y^{\alpha} \mid \alpha \in N\right\} \subseteq C[n, m], \\
& Z=\operatorname{span}(A) \leq C[n, m] .
\end{aligned}
$$

By inspecting the generators and relations on both sides, we see that

$$
C[n, m] /\left(C[n, m] y_{k-1}\right)=\bar{C}[n, k-1] \otimes C[k, m] .
$$

Propositions 7.5 and 7.9 show that $A$ also gives a basis for $C[n, m] /\left(C[n, m] y_{k-1}\right)$, so $C[n, m]=Z \oplus\left(C[n, m] y_{k-1}\right)$. Now let $g$ denote the composite

$$
Z \stackrel{\simeq}{\rightarrow} C[n, m] /\left(C[n, m] y_{k-1}\right) \stackrel{f}{\rightarrow} C[n, m] \stackrel{\text { proj }}{\rightarrow} C[n, m] / Z .
$$

It will certainly be enough to show that $g$ is injective. It is not hard to see that $y_{k} Z \leq Z$, and $x_{k}=y_{k-1}^{2}+y_{k}$, so $g(z)=x_{k} z+Z=y_{k-1}^{2} z+Z$, and so $g$ gives an injective map from $A$ to $B C[n, m] \backslash A$. These sets are bases for the domain and codomain of $g$, so $g$ is injective as required.

Proposition 7.18. $C$ is self-injective.

Proof. As $C$ is finite in each degree, it will suffice (by Propositions 3.3 and 3.6) to show that every test pair $(u, v)$ in $C$ has either a block or a transporter. Let $d$ be the degree of $(u, v)$, so $\left|v_{i}\right|=\left|u_{i}\right|+d$. Note that some of the entries $u_{i}$ and $v_{i}$ may be zero, in which case $\left|u_{i}\right|$ or $\left|v_{i}\right|$ can be negative. Choose $m$ such that $2^{m}>d$ and also $2^{m}>\left|u_{i}\right|$ and $2^{m}>\left|v_{i}\right|$ for all $i$. Now $(u, v)$ can be regarded as a test pair in $C[n, m]$. Let $\pi$ be the projection $C[n, m] \rightarrow \bar{C}[n, m]=C[n, m] / y_{m}$. As $\bar{C}[n, m]$ has Poincaré duality, it is self-injective, so the test pair $(\pi(u), \pi(v))$ has either a block or a transporter. First, suppose that there is a transporter $\pi(t)$, so $\pi\left(v_{i}\right)=\pi\left(t u_{i}\right)$ for all $i$. This is an equation between elements of degree $\left|v_{i}\right|<2^{m}$, and $\pi: C[n, m] \rightarrow \bar{C}[n, m]$ is an isomorphism in this degree, so $v_{i}=t u_{i}$, so we have a transporter for the original pair $(u, v)$.

Suppose instead that there is a block for $(\pi(u), \pi(v))$, say $\pi(b)$. This means that $\pi(b . u)=0$ but $\pi(b . v) \neq 0$, so $b . u \in C[n, m] y_{m}$ but $b . v \notin C[n, m] y_{m}$. Using our bases for the various rings under consideration, we see that $C[n, m] y_{m}=$ $\left(C y_{m}\right) \cap C[n, m]$, and thus that $b . v \notin C y_{m}$. It now follows from Proposition 7.17 that $\left(x_{m+1} b\right) \cdot u=0$ and $\left(x_{m+1} b\right) \cdot v \neq 0$, so $x_{m+1} b$ is a block for the original pair $(u, v)$. 
We now wish to prove that $C$ is coherent, which turns out to involve substantial work. It will be convenient to regard the set $B \bar{C}[n, m]=\left\{y^{\alpha} \mid \alpha \in M \bar{C}[n, m]\right\}$ as a subset of $C[n, m]$ rather than a subset of $\bar{C}[n, m]$. We write $\tilde{C}[n, m]$ for the span of this set, so the projection $C[n, m] \rightarrow \bar{C}[n, m]$ restricts to give an isomorphism $\tilde{C}[n, m] \rightarrow \bar{C}[n, m]$.

Lemma 7.19. For $p \geq 3$ we have

$$
y_{[0, p-3]}^{2} y_{[0, p-1]}^{2} y_{1} y_{p-1} y_{p}=y_{[0, p]}^{2}
$$

(and in particular, this is nonzero modulo $y_{p+1}$ ).

Proof. Put $A=C[0, p] / \operatorname{ann}\left(y_{[0, p]}\right)$. We claim that in $A$ we have

$$
y_{[0, p-3]}^{2} y_{[0, p-1]} y_{1} y_{p-1}=y_{[0, p]} .
$$

Assuming this, we can just multiply by $y_{[0, p]}$ to recover the statement in the lemma.

For $0 \leq i<p$ we have $y_{i}\left(y_{i}^{2}+y_{i+1}\right)=0$ so $y_{[0, p]}\left(y_{i}^{2}+y_{i+1}\right)=0$ and so $y_{i+1}=y_{i}^{2}$ in $A$. We thus have $y_{k}=y_{0}^{2^{k}}$ in $A$ for $0 \leq k \leq p$, and so $A=\mathbb{F}\left[y_{0}\right]$. It is thus enough to show that the two sides of the claimed equation have the same degree, which is a straightforward calculation.

Lemma 7.20. For any $p \geq 3$ we have

$$
B \bar{C}[0, p-2] B \bar{C}[0, p] \subseteq \coprod_{i=0}^{3} B \bar{C}[0, p-1] y_{p-1}^{i} .
$$

Proof. Consider $\alpha \in M \bar{C}[0, p-2]$ and $\beta \in M \bar{C}[0, p]$. We note that

$$
y^{\alpha}, y^{\beta} \in C[0, p-1]
$$

so we can rewrite $y^{\alpha+\beta}$ as an element of the basis $B^{\prime} C[0, p-1]$, which means $y^{\alpha+\beta}=y^{\gamma}$ for some $\gamma \in M^{\prime} C[0, p-1]$. It will be enough to show that $\gamma_{p-1} \leq 3$.

Note that $y^{\alpha}$ divides $y_{[0, p-3]}^{2}$ and $y^{\beta}$ divides $y_{[0, p-1]}^{2}$ so $y^{\gamma}$ divides $y_{[0, p-3]}^{2} y_{[0, p-1]}^{2}$. It follows using Lemma 7.19 that $y^{\gamma} y_{p-1} y_{p} \neq 0\left(\bmod y_{p+1}\right)$. However,

$$
y_{p-1}^{4} y_{p-1} y_{p}=y_{p-1}^{5} y_{p}=y_{p-1}^{3} y_{p}^{2}=y_{p-1} y_{p}^{3}=y_{p-1} y_{p} y_{p+1}=0\left(\bmod y_{p+1}\right),
$$

so $y^{\gamma}$ cannot be divisible by $y_{p-1}^{4}$, as required.

Definition 7.21. For any vector $u \in C^{n}$ and $p \geq 0$, we put

$$
\begin{aligned}
& K(u, p)=\left\{v \in C[0, p]^{n} \mid u \cdot v=0\right\}, \\
& \bar{K}(u, p)=\left\{\bar{v} \in \bar{C}[0, p]^{n} \mid \pi(u) \cdot \bar{v}=0\right\} .
\end{aligned}
$$


More precisely, $K(u, p)$ is the graded group where

$$
K(u, p)_{d}=\left\{v \in C[0, p]^{n}|| v_{i}|=d-| u_{i} \mid \text { for all } i \text { and } \sum_{i} u_{i} v_{i}=0\right\},
$$

and $\bar{K}(u, p)$ is graded in a similar way.

Lemma 7.22. If $u_{i} \in \tilde{C}[0, p-2]$ for all $i$, then the map

$$
\pi: K(u, p+1) \rightarrow \bar{K}(u, p+1)
$$

is surjective.

Proof. Consider an element $\bar{v} \in \bar{K}(u, p+1)$. This can be written as $\pi(v)$ for a unique element $v \in \tilde{C}[0, p+1]^{n}$, which must satisfy $u . v=0\left(\bmod y_{p+1}\right)$. We can write $v$ as $\sum_{k=0}^{2} v_{k} y_{p}^{k}$ with $v_{k} \in \tilde{C}[0, p]^{n}$. Using Lemma 7.20 we see that $u . v_{k}$ can be written as $\sum_{j=0}^{3} w_{j k} y_{p-1}^{j}$ for some elements $w_{j k} \in \tilde{C}[0, p-1]$. This gives $u . v=\sum_{j=0}^{3} \sum_{k=0}^{2} w_{j k} y_{p-1}^{j} y_{p}^{k}$. After reducing the terms $y_{p-1}^{j} y_{p}^{k}$ using the defining relations for $C$, we obtain

$$
\begin{array}{r}
u . v=w_{00}+w_{01} y_{p}+w_{02} y_{p}^{2}+w_{10} y_{p-1}+\left(w_{11}+w_{30}\right) y_{p-1} y_{p}+\left(w_{12}+w_{31}\right) y_{p-1} y_{p}^{2} \\
+w_{20} y_{p-1}^{2}+w_{21} y_{p-1}^{2} y_{p}+w_{22} y_{p-1}^{2} y_{p}^{2}+w_{32} y_{p-1} y_{p} y_{p+1} .
\end{array}
$$

By hypothesis, this maps to zero in $\bar{C}[0, p+1]=C[0, p+1] / y_{p+1}$. However, $\bar{C}[0, p+1]$ splits as the direct sum of subgroups $\tilde{C}[0, p-1] y_{p-1}^{i} y_{p}^{j}$ for $0 \leq i, j<3$, so we must have

$$
w_{00}=w_{01}=w_{02}=w_{10}=w_{20}=w_{21}=w_{22}=0
$$

and $w_{11}=w_{30}$ and $w_{12}=w_{31}$, so $u . v=w_{32} y_{p-1} y_{p} y_{p+1}$.

Now put $d=|u . v|$, so $\left|w_{j k}\right|=d-j 2^{p-1}-k 2^{p}$. In particular, we have

$$
\left|w_{32}\right|=d-2^{p-1}-2^{p}-2^{p+1} .
$$

If $d<2^{p-1}+2^{p}+2^{p+1}$ then $\left|w_{32}\right|<0$ so $w_{32}=0$, and so $u . v=0$. This means that $v \in K(u, p+1)$ with $\pi(v)=\bar{v}$, as required. Suppose instead that $d \geq 2^{p-1}+2^{p}+2^{p+1}$. We have

$$
\begin{aligned}
& \left|w_{11}\right|=\left|w_{30}\right|=d-2^{p-1}-2^{p} \geq 2^{p+1}, \\
& \left|w_{12}\right|=\left|w_{31}\right|=d-2^{p-1}-2^{p+1} \geq 2^{p} .
\end{aligned}
$$

However, the elements $w_{j k}$ lie in $\tilde{C}[0, p-1]$, which is zero in degrees larger than $2^{p}-2$. We therefore have $w_{11}=w_{12}=w_{30}=w_{31}=0$, which means that $u \cdot v_{0}=0$ and $u \cdot v_{1}=0$ and $u \cdot v_{2}=w_{32} y_{p-1}^{3}=w_{32} y_{p-1} y_{p}$. Put

$$
v^{\prime}=v_{0}+v_{1} y_{p}+v_{2}\left(y_{p}^{2}+y_{p+1}\right)
$$


so $\pi\left(v^{\prime}\right)=\pi(v)=\bar{v}$ and

$$
u . v^{\prime}=u \cdot v_{0}+u \cdot v_{1} y_{p}+u \cdot v_{2}\left(y_{p}^{2}+y_{p+1}\right)=w_{32} y_{p-1} y_{p}\left(y_{p}^{2}+y_{p+1}\right)=0 .
$$

Thus, $v^{\prime}$ is the required lift of $\bar{v}$ in $\bar{K}(u, p+1)$.

Lemma 7.23. For all $p \geq 0$ we have a splitting

$$
C[0, p+1]=C[0, p] \oplus \bigoplus_{k>0} \bar{C}[0, p] x_{p+1}^{k} .
$$

Proof. By definition, we have $C[0, p+1]=C[0, p]\left[y_{p+1}\right] /\left(x_{p+1} y_{p}\right)$, where $x_{p+1}=y_{p+1}+y_{p}^{2}$ as usual. From this it is clear that

$$
C[0, p]\left[y_{p+1}\right]=C[0, p]\left[x_{p+1}\right]=C[0, p] \oplus \bigoplus_{k>0} C[0, p] x_{p+1}^{k} .
$$

The ideal generated by $y_{p} x_{p+1}$ in this ring clearly has a compatible splitting

$$
C[0, p]\left[y_{p+1}\right] \cdot y_{p} x_{p+1}=\bigoplus_{k>0} C[0, p] y_{p} x_{p+1}^{k} \text {. }
$$

We can thus pass to the quotient to get

$$
C[0, p+1]=C[0, p] \oplus \bigoplus_{k>0} \frac{C[0, p]}{C[0, p] y_{p}} x_{p+1}^{k}=C[0, p] \oplus \bigoplus_{k>0} \bar{C}[0, p] x_{p+1}^{k}
$$

as claimed.

Corollary 7.24. If $u_{i} \in \tilde{C}[0, p-2]$ for $i=0, \ldots, n-1$, then

$$
K(u, p+1)=C[0, p+1] \cdot K(u, p) .
$$

Proof. It is clear that $C[0, p+1] . K(u, p) \leq K(u, p+1)$. For the converse, consider an element $v \in K(u, p+1) \leq C[0, p+1]^{n}$. Using Lemma 7.23, we can write $v$ as $v_{0}+\sum_{k>0} \bar{v}_{k} x_{p+1}^{k}$, with $v_{0} \in C[0, p]^{n}$ and $\bar{v}_{k} \in \bar{C}[0, p]^{n}$ (with $\bar{v}_{k}=0$ for $k \gg 0$ ). It follows that $u . v_{0} \in C[0, p]$ and $u . \bar{v}_{k} \in \bar{C}[0, p]$ and

$$
u . v_{0}+\sum_{k>0}\left(u . \bar{v}_{k}\right) x_{p+1}^{k}=u . v=0 .
$$

As the sum in Lemma 7.23 is direct, we must have $u . v_{0}=0$ and $u \cdot \bar{v}_{k}=0$, so $v_{0} \in K(u, p)$ and $\bar{v}_{k} \in \bar{K}(u, p)$. By Lemma 7.22, we can choose $v_{k} \in K(u, p)$ for $k>0$ lifting $\bar{v}_{k}$. If $\bar{v}_{k}=0$ we choose $v_{k}=0$; this ensures that $v_{k}=0$ for $k \gg 0$. We now have $v=\sum_{k \geq 0} v_{k} x_{p+1}^{k} \in C[0, p+1] . K(u, p)$, as required.

Proposition 7.25. The ring $C$ is coherent. 
Proof. Let $I \leq C$ be a finitely generated ideal. Choose elements $u_{0}, \ldots, u_{n-1}$ generating $I$. These give an epimorphism $g: \bigoplus_{i} \Sigma^{\left|u_{i}\right|} C \rightarrow I$, with $\operatorname{ker}(g)=$ $K(u, \infty)$, so it will suffice to show that $K(u, \infty)$ is finitely generated as a $C$-module. Now choose $p$ large enough that $u_{i} \in \tilde{C}[0, p-2]$ for all $i$. As $C[0, p]$ is Noetherian, we can choose a finite subset $T \subseteq C[0, p]^{n}$ that generates $K(u, p)$ as a $C[0, p]$ module. Corollary 7.24 tells us that $T$ also generates $K(u, p+1)$ as a $C[0, p+1]$ module. In fact, we can apply the same corollary inductively to see that $T$ generates $K(u, q)$ as a $C[0, q]$-module for all $q \geq p$. As $C=\bigcup_{q} C[0, q]$ we conclude that $T$ generates $K(u, \infty)$ as required.

Proposition 7.26. The reduced quotient of $C$ is

$$
C / \sqrt{0}=\mathbb{F}\left[x_{i} \mid i \geq 0\right] /\left(x_{i} x_{j} \mid i \neq j\right) .
$$

Proof. Put $C^{\prime}=C / \sqrt{0}$. We first claim that for all $p, q$ with $0 \leq p<q$ we have $x_{p} x_{q}=0$ in $C^{\prime}$. We may assume inductively that $x_{i} x_{j}=0$ in $C^{\prime}$ whenever $0 \leq i<j<q$. By a nested downward induction over $p$, we may assume that $x_{k} x_{q}=0$ in $C^{\prime}$ whenever $p<k<q$. As in Proposition 7.12, we have $x_{q} \sum_{k=0}^{q-1} x_{k}^{2^{q-1-k}}=0$. We can multiply this by $x_{p}$ and use the inner and outer inductive assumptions to see that $x_{p} x_{q} x_{p}^{2^{q-1-p}}=0$, or in other words $x_{p}^{m} x_{q}=0$ for some $m>0$. This gives $\left(x_{p} x_{q}\right)^{m}=0$ in $C^{\prime}$, but $C^{\prime}$ is reduced by construction so $x_{p} x_{q}=0$ in $C^{\prime}$ as claimed.

Now put

$$
C^{\prime \prime}=C /\left(x_{i} x_{j} \mid i, j, i<j\right)=\mathbb{F}\left[x_{i} \mid i \geq 0\right] /\left(x_{i} x_{j} \mid i, j \geq 0, i<j\right) .
$$

We now see that $C^{\prime \prime}$ is a quotient of $C$ by nilpotent elements, so $C^{\prime}$ can also be described as $C^{\prime \prime} / \sqrt{0}$. However, there is an obvious splitting

$$
C^{\prime \prime}=\mathbb{F} \oplus \bigoplus_{i \geq 0} x_{i} \mathbb{F}\left[x_{i}\right],
$$

and using this we see that $C^{\prime \prime}$ is reduced. It follows that $C^{\prime}=C^{\prime \prime}$ as claimed.

\section{Pontrjagin self-dual rings}

Let $R$ be a Pontrjagin self-dual ring, as in Definition 1.7. Thus, $R$ is a graded $\mathbb{Z}_{p}$-algebra $R$ equipped with an isomorphism $\zeta: R_{d} \rightarrow \mathbb{Q}_{p} / \mathbb{Z}_{p}$ (for some $d$ ) such that the resulting maps

$$
\zeta^{\#}: R_{d-k} \rightarrow R_{k}^{\vee}=\operatorname{Hom}_{\mathbb{Z}_{p}}\left(R_{k}, \mathbb{Q}_{p} / \mathbb{Z}_{p}\right)
$$

are isomorphisms.

Lemma 8.1. For graded $R$-modules $M$ there is a natural isomorphism

$$
\operatorname{Hom}_{R}(M, R) \simeq \operatorname{Hom}_{\mathbb{Z}_{p}}\left(M_{d}, \mathbb{Q}_{p} / \mathbb{Z}_{p}\right)=M_{d}^{\vee}
$$


Proof. Given $\phi \in \operatorname{Hom}_{R}(M, R)$, we put

$$
\tau(\phi)=\zeta \circ \phi_{d}: M_{d} \rightarrow \mathbb{Q}_{p} / \mathbb{Z}_{p} .
$$

This defines a map $\tau: \operatorname{Hom}_{R}(M, R) \rightarrow M_{d}^{\vee}$.

Now suppose we have a map $\psi: M_{d} \rightarrow \mathbb{Q}_{p} / \mathbb{Z}_{p}$. For any $k \in \mathbb{Z}$ we have a map

$$
\phi_{k}^{\prime}: M_{k} \rightarrow \operatorname{Hom}_{\mathbb{Z}_{p}}\left(R_{d-k}, \mathbb{Q}_{p} / \mathbb{Z}_{p}\right)
$$

given by $\phi_{k}^{\prime}(m)(a)=(-1)^{k(d-k)} \psi(a m)$. As $R$ is assumed to be Pontrjagin self-dual, there is a unique element $\phi_{k}(m) \in R_{k}$ such that

$$
\phi_{k}^{\prime}(m)(a)=\zeta\left(\phi_{k}(m) a\right)
$$

for all $a \in R_{d-k}$. We leave it to the reader to check that this gives a map $\phi: M \rightarrow R$ of $R$-modules, and that this is the unique such map with $\tau(\phi)=\psi$.

Proposition 8.2. Any Pontrjagin self-dual ring is self-injective.

Proof. We need to show that the functor $M \mapsto \operatorname{Hom}_{R}(M, R)$ is exact, but it is isomorphic to the functor $M \mapsto \operatorname{Hom}_{\mathbb{Z}_{p}}\left(M_{d}, \mathbb{Q}_{p} / \mathbb{Z}_{p}\right)$, which is exact because $\mathbb{Q}_{p} / \mathbb{Z}_{p}$ is divisible and therefore injective as a $\mathbb{Z}_{p}$-module.

We now study the graded ring $J$ described by Definition 1.9, and the tensor product $\hat{J}=\mathbb{Z}_{p} \otimes J$. It is standard that $\mathbb{Z}_{p} \otimes \mathbb{Z} / p^{r}=\mathbb{Z} / p^{r}$. Moreover, the group $\mathbb{Q}_{p} / \mathbb{Z}_{p}$ can be written as the colimit of the evident sequence

$$
\mathbb{Z} / p \rightarrow \mathbb{Z} / p^{2} \rightarrow \mathbb{Z} / p^{3} \rightarrow \cdots,
$$

and we can tensor with $\mathbb{Z}_{p}$ to get $\mathbb{Z}_{p} \otimes\left(\mathbb{Q}_{p} / \mathbb{Z}_{p}\right)=\mathbb{Q}_{p} / \mathbb{Z}_{p}$. Thus, the only difference between $J$ and $\hat{J}$ is that $J_{0}=\mathbb{Z}_{(p)}$ whereas $\hat{J}_{0}=\mathbb{Z}_{p}$.

Lemma 8.3. The ring $\hat{J}$ is Pontrjagin self-dual, so $\hat{J}_{-2-k} \simeq \hat{J}_{k}^{\vee}$.

Proof. For $k \neq-2$ this is a straightforward calculation. For $k=-2$ we use the description $\mathbb{Q}_{p} / \mathbb{Z}_{p}=\lim _{j} \mathbb{Z} / p^{j}$ to get

as required.

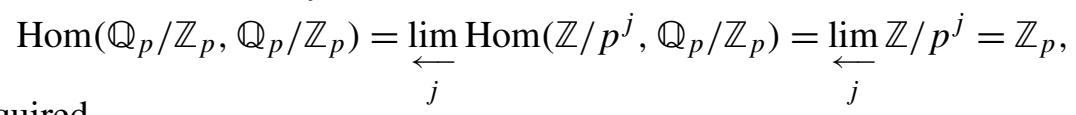

Corollary 8.4. The ring $\hat{J}$ is self-injective.

Remark 8.5. The ring $J$ itself is not self-injective. To see this, note that $J_{-2}$ is an ideal in $J$ and is a module over $\mathbb{Z}_{p}$. Choose any element $a \in \mathbb{Z}_{p} \backslash \mathbb{Z}_{(p)}$ and define $u: J_{-2} \rightarrow J$ by $u(x)=a x$. This cannot be extended to give a $J$-linear endomorphism of $J$. 
Lemma 8.6. The ring $\hat{J}$ is local (in the graded sense). The unique maximal graded ideal is given by $\mathfrak{m}_{0}=p \mathbb{Z}_{p}$ and $\mathfrak{m}_{k}=\hat{J}_{k}$ for all $k \neq 0$. Moreover, the elements $\alpha_{k}$ together with the element $p$ give a basis for $\mathfrak{m} / \mathfrak{m}^{2}$ over $\mathbb{Z} / p$.

Proof. It is straightforward to check that the graded group $\mathfrak{m}$ described above is an ideal in $\hat{J}$, and the quotient $\hat{J} / \mathfrak{m}$ is the field $\mathbb{Z} / p$, so it is a maximal ideal. Let $\mathfrak{m}^{\prime}$ be an arbitrary maximal graded ideal. Put $\mathfrak{a}=\bigoplus_{k \neq 0} \hat{J}_{k}$. Every homogeneous element $a \in \mathfrak{a}$ satisfies $a^{2}=0$, and it follows that $\mathfrak{a} \leq \mathfrak{m}^{\prime}$ This means that $\mathfrak{m}^{\prime}$ corresponds to a maximal ideal in the quotient $\hat{J} / \mathfrak{a} \simeq \mathbb{Z}_{p}$, and the only such ideal is $p \mathbb{Z}_{p}$. It follows that $\mathfrak{m}^{\prime}=\mathfrak{m}$ as claimed. The description of $\mathfrak{m} / \mathfrak{m}^{2}$ is a straightforward calculation.

Proposition 8.7. The ring $\hat{J}$ is totally incoherent.

Proof. Put $V=\left\{\alpha_{k} \mid k \neq 0(\bmod p)\right\} \subset J$, so $V$ is infinite, $p V$ vanishes and $V$ remains linearly independent in $\mathfrak{m} / \mathfrak{m}^{2}$. By inspecting the multiplication rules, we see that every noninvertible element of $\hat{J}$ annihilates all elements of $V$ with at most one exception. It follows using Corollary 5.6 that $\hat{J}$ is totally incoherent.

\section{The infinite root algebra}

In this section we fix a field $K$ and study the infinite root algebra $P$ over $K$, which was introduced in Definition 1.12. We first recall the details.

Definition 9.1. We say that a subset $U \subseteq[0,1]$ is well-ordered if the usual order inherited from $\mathbb{R}$ is a well-ordering, so every nonempty subset of $U$ has a smallest element. It is equivalent to say that every infinite nonincreasing sequence in $U$ is eventually constant, or that there are no infinite, strictly decreasing sequences.

An infinite root series is a function $a:[0,1] \rightarrow K$ such that the $\operatorname{set} \operatorname{supp}(a)=$ $\{q \mid a(q) \neq 0\}$ is well-ordered. The infinite root algebra is the set $P$ of all infinite root series. We regard this as an ungraded object, or equivalently as a graded object concentrated in degree zero.

Remark 9.2. It is clear that any subset of a well-ordered set is well-ordered, and that the union of any two well-ordered sets is well-ordered. Now if $a, b \in P$ we have $\operatorname{supp}(a+b) \subseteq \operatorname{supp}(a) \cup \operatorname{supp}(b)$, so $P$ is closed under addition. It is clearly also closed under multiplication by elements of $K$.

Lemma 9.3. Any well-ordered subset of $[0,1]$ is countable. Moreover, for any countable ordinal $\alpha$, there is a well-ordered subset $U \subseteq[0,1]$ that is orderisomorphic to $\alpha$.

Proof. Firstly, we can regard rational numbers in $[0,1]$ as coprime pairs of integers and this gives a lexicographic ordering on $\mathbb{Q} \cap[0,1]$, which is a well-ordering. 
Next, let $U$ be a well-ordered subset of $[0,1]$. We define $f: U \rightarrow \mathbb{Q}$ as follows. If $u$ is maximal in $U$, we put $f(u)=1$. Otherwise, the set $\{v \in U \mid v>u\}$ has a smallest element $v_{0}$, and we define $f(u)$ to be the lexicographically smallest element of $\mathbb{Q} \cap\left[u, v_{0}\right)$. It is clear that $f$ is injective, so $U$ is countable.

Let $\alpha$ be any countable ordinal; we claim that there is an order-embedding $g: \alpha \rightarrow[0,1]$. To see this, choose an injective map $p: \alpha \rightarrow \mathbb{N}$ and then put

$$
g(\beta)=\sum_{\gamma<\beta} 2^{-p(\gamma)-1} .
$$

It is clear that this has the required properties.

Lemma 9.4. If $U, V \subseteq[0,1]$ are well-ordered and $w \in[0,1]$, then

$$
\{(u, v) \in U \times V \mid u+v=w\}
$$

is finite.

Proof. Put $U^{\prime}=\{u \in U \mid w-u \in V\}$. This is well-ordered (because it is a subset of $U$ ) and it will suffice to show that it is finite. If not, we can define an infinite sequence $u_{0}<u_{1}<u_{2}<\cdots$ in $U^{\prime}$ as follows: we take $u_{0}$ to be the smallest element in $U^{\prime}$, then take $u_{1}$ to be the smallest element in $U^{\prime} \backslash\left\{u_{0}\right\}$, and so on. We then note that $w-u_{0}, w-u_{1}, w-u_{2}, \ldots$ is an infinite strictly decreasing sequence in $V$, contradicting the assumption that $V$ is well-ordered.

Lemma 9.5. Let $U$ be a well-ordered subset of $[0,1]$, and let $\left(u_{n}\right)$ be a sequence in $U$. Then there exists an infinite nondecreasing subsequence.

Proof. Put $v_{0}=\min \left\{u_{j} \mid j \geq 0\right\}$ (which is meaningful because $U$ is well-ordered) and then $n_{0}=\min \left\{j \mid u_{j}=v_{0}\right\}$. For $i>0$, we define recursively

$$
v_{i}=\min \left\{u_{j} \mid j>n_{i-1}\right\} \quad \text { and } \quad n_{i}=\min \left\{j>n_{i-1} \mid u_{j}=v_{i}\right\} .
$$

We find that $n_{0}<n_{1}<n_{2}<\cdots$ and $v_{0} \leq v_{1} \leq v_{2} \leq \cdots$, or equivalently that $u_{n_{0}} \leq u_{n_{1}} \leq u_{n_{2}} \leq \cdots$ as required.

Lemma 9.6. Let $U$ and $V$ be well-ordered subsets of [0,1], and put

$$
U * V=\{u+v \mid u \in U \text { and } v \in V\} .
$$

Then $U * V$ is also well-ordered.

Proof. Suppose not. We can then find an infinite strictly descending chain in $U * V$, so we can choose a sequence $\left(u_{n}, v_{n}\right)$ in $U \times V$ with $u_{i}+v_{i}>u_{i+1}+v_{i+1}$ for all $i$. Lemma 9.5 tells us that after passing to a subsequence, we may assume that $u_{j} \leq u_{j+1}$ for all $j$. After passing again to a sparser subsequence, we may also assume that $v_{k} \leq v_{k+1}$ for all $k$. This is clearly impossible. 
Proposition 9.7. We can make $P$ into a commutative ring by the rule

$$
a b(w)=\sum_{w=u+v} a(u) b(v) .
$$

Proof. Lemma 9.4 shows that the sum is essentially finite, so there is no problem with convergence. It is clear that $\operatorname{supp}(a b) \subseteq \operatorname{supp}(a) * \operatorname{supp}(b)$, and Lemma 9.6 shows that $\operatorname{supp}(a) * \operatorname{supp}(b)$ is well-ordered, so $a b \in P$. It is straightforward to check that the multiplication operation is commutative, associative and bilinear. Moreover, if we define $e(0)=1$ and $e(q)=0$ for $q \neq 0$, then $e$ is a multiplicative identity element for $P$.

Definition 9.8. For $a \in P \backslash\{0\}$, we put $\delta(a)=\min (\operatorname{supp}(a))$. We also put $\delta(0)=\infty$.

Remark 9.9. If $\delta(a)+\delta(b) \leq 1$ we have

$$
(a b)(\delta(a)+\delta(b))=a(\delta(a)) b(\delta(b)) \neq 0,
$$

so $a b \neq 0$ and $\delta(a b)=\delta(a)+\delta(b)$. On the other hand, if $\delta(a)+\delta(b)>1$ then $a b=0$.

Definition 9.10. For $q \in \mathbb{R} \cup\{\infty\}$ with $q \geq 0$, we define $x^{q} \in P$ by

$$
x^{q}(u)= \begin{cases}1 & \text { if } u=q, \\ 0 & \text { otherwise. }\end{cases}
$$

Remark 9.11. (a) $x^{0}$ is the multiplicative identity element $e$.

(b) If $q>1$ then $x^{q}=0$.

(c) If $0 \leq q \leq 1$ then $\delta\left(x^{q}\right)=q$.

(d) For all $q, r \geq 0$ we have $x^{q} x^{r}=x^{q+r}$.

Lemma 9.12. Consider an element $a \in P \backslash\{0\}$. If $a(0)=0$ (or equivalently, $\delta(a)>0)$ then $a$ is nilpotent, but if $\delta(a)=0$ then a is invertible.

Proof. If $\delta(a)>0$ then we can find a positive integer $n$ with $\delta(a)>1 / n$, and using Remark 9.9 we see that $a^{n}=0$. Suppose instead that $\delta(a)=0$. We can then write $a=u e+b=u(e+b / u)$, where $u \in K \backslash 0$ and $e=x^{0}$ is the multiplicative identity of $P$ and $\delta(b)>0$, so $b^{n}=0$ for some $n$. Now $a$ has inverse $\sum_{i=0}^{n-1} u^{-1}(-b / u)^{i}$.

Corollary 9.13. The map $a \mapsto a(0)$ induces an isomorphism $P / \sqrt{0} \rightarrow K$.

Proof. Clear.

Definition 9.14. For $a \in P$ with $\delta(a) \geq t$, we define $\lambda_{t}(a) \in P$ by

$$
\lambda_{t}(a)(r)= \begin{cases}a(r+t) & \text { if } 0 \leq r \leq 1-t, \\ 0 & \text { if } 1-t<r \leq 1 .\end{cases}
$$


Corollary 9.15. If $\delta(a) \geq t$ then $a=x^{t} \lambda_{t}(a)$ and $\delta\left(\lambda_{t}(a)\right)=\delta(a)-t$. Moreover, if $\delta(a)=t$ then $\lambda_{t}(a)$ is invertible, so $P a=P x^{t}$.

Proof. The first two claims are clear from the definitions, and the third then follows using Lemma 9.12.

Definition 9.16. For $t \in[0,1]$ we put

$$
\begin{aligned}
& J_{t}=\{a \in P \mid \delta(a)>t\}, \\
& \bar{J}_{t}=\{a \in P \mid \delta(a) \geq t\}=P x^{t} .
\end{aligned}
$$

Proposition 9.17. Every ideal in $P$ has the form $J_{t}$ or $\bar{J}_{t}$.

Proof. Let $I$ be an ideal in $P$. If $I=0$ then $I=J_{1}$. Otherwise, we put $t=\inf \{\delta(a) \mid a \in I\}$. If $t=\delta(a)$ for some $a \in I$ then Corollary 9.15 shows that $x^{t} \in I$, and it follows easily that $I=\bar{J}_{t}$. Suppose instead that there is no element $a \in I$ with $\delta(a)=t$. It is then clear that $I \leq J_{t}$. Moreover, if $b \in J_{t}$ then $\delta(b)>t$, so (by the infimum condition) there exists $a \in I$ with $\delta(b)>\delta(a)>t$. After applying Corollary 9.15 to $a$ and $b$, we see that $b$ is a multiple of $a$, and so $b \in I$. We now see that $I=J_{t}$, as required.

Proposition 9.18. For all $t \in[0,1]$ we have $\operatorname{ann}_{P}\left(J_{t}\right)=\bar{J}_{1-t}$ and $\operatorname{ann}_{P}\left(\bar{J}_{t}\right)=J_{1-t}$. Proof. This follows easily from the fact that $a b=0$ if and only if $\delta(a)+\delta(b)>1$.

Corollary 9.19. For any ideal $I \leq P$ we have $\operatorname{ann}_{P}^{2}(I)=I$.

Proof. Immediate from the last two propositions.

Proposition 9.20. $P$ is self-injective.

Proof. As we have classified all ideals in $P$, we can use Baer's criterion. Consider a number $t \in[0,1]$ and a $P$-module map $f: \bar{J}_{t}=\left(x^{t}\right) \rightarrow P$. If $f\left(x^{t}\right)=a$ then we must have $J_{1-t} a=f\left(J_{1-t} x^{t}\right)=f(0)=0$, so $a \in \operatorname{ann}\left(J_{1-t}\right)=\bar{J}_{t}$, so $a=x^{t} \lambda_{t}(a)$. We can now define $f^{\prime}: P \rightarrow P$ extending $f$ by $f^{\prime}(p)=p \lambda_{t}(a)$, so Baer's criterion is satisfied in this case.

Now consider instead a $P$-module map $f: J_{t} \rightarrow P$. If $t=1$ then $J_{t}=0$ and the zero map $P \rightarrow P$ extends $f$. We suppose instead that $t<1$. For $s \in(t, 1]$ we put $a_{s}=\lambda_{s}\left(f\left(x^{s}\right)\right)$, so the first case shows that $f(p)=p a_{s}$ for all $p \in \bar{J}_{s}<J_{t}$. Now suppose that $t<r \leq s \leq 1$. As $x^{s} \in \bar{J}_{s} \leq \bar{J}_{r}$ we have $x^{s}\left(a_{r}-a_{s}\right)=f\left(x^{s}\right)-f\left(x^{s}\right)=0$, so $a_{r}(q)=a_{s}(q)$ for all $q \leq 1-s$. Moreover, from the definition of the $\lambda$ operation we have $a_{s}(q)=0$ for $q>1-s$, and thus certainly for $q \geq 1-t$. We now see that there is a unique map $a:[0,1] \rightarrow K$ with $a=a_{s}$ on $[0,1-s]$ (for all $s \in(t, 1]$ ) and $a=0$ on $[1-t, 1]$. It follows easily from these properties that $\operatorname{supp}(a)$ is well-ordered, so $a \in P$. We also see from the first property that $f$ agrees with multiplication by $a$ on $\bar{J}_{s}$ for all $s \in(t, 1]$. It follows that the same is true on $\bigcup_{s \in(t, 1]} \bar{J}_{s}=J_{t}$, as required. 


\section{Proposition 9.21. $P$ is totally incoherent.}

Proof. Let $I$ be a finitely generated ideal, say $I=\left(a_{1}, \ldots, a_{r}\right)$, where we can assume that the generators $a_{i}$ are nonzero. If $r=0$ then $I=0$, and this is finitely presented. If $r>0$ we can use Corollary 9.15 to see that $I=\bar{J}_{t}$, where $t=\min \left(\delta\left(a_{1}\right), \ldots, \delta\left(a_{r}\right)\right)$.

Now suppose that $I$ is nonzero and finitely presented. We must have $I=\bar{J}_{t}$ for some $t$, so we have an epimorphism $g: P \rightarrow I$ given by $g(a)=a x^{t}$. Definition 5.1 tells us that $\operatorname{ker}(g)$ must also be finitely generated, but $\operatorname{ker}(g)=\operatorname{ann}_{P}\left(x^{t}\right)=J_{1-t}$, and this is only finitely generated when $t=0$ and $\operatorname{so} \operatorname{ker}(g)=J_{1}=0$ and $I=\bar{J}_{0}=P$.

Remark 9.22. Put $P^{\prime}=\{a \in P \mid \operatorname{supp}(a) \subseteq \mathbb{Q}\}$. This is a subring of $P$, and one can adapt the above arguments to show that it is again self-injective and totally incoherent. Every ideal in $P^{\prime}$ has the form $J_{t} \cap P^{\prime}$ or $\bar{J}_{t} \cap P^{\prime}$ for some $t \in[0,1]$, and these are all distinct except for the fact that $J_{t} \cap P^{\prime}=\bar{J}_{t} \cap P^{\prime}$ when $t$ is irrational.

\section{The Rado algebra}

In this section we study the Rado algebra $Q$, which was defined in Definition 1.16. We will write $\Gamma$ for the Rado graph.

We first clarify the kinds of graphs that we will consider.

Definition 10.1. A graph is a pair $(V, E)$, where $V$ is a set and $E$ is a subset of $V \times V$ such that:

(a) For all $v \in V$ we have $(v, v) \notin E$.

(b) For all $v, w \in V$ we have $((v, w) \in E$ if and only if $(w, v) \in E)$.

Definition 10.2. Let $G=(V, E)$ and $G^{\prime}=\left(V^{\prime}, E^{\prime}\right)$ be graphs. A full embedding of $G$ in $G^{\prime}$ is an injective map $f: V \rightarrow V^{\prime}$ such that $E=(f \times f)^{-1}\left(E^{\prime}\right)$ (so vertices $v_{0}, v_{1} \in V$ are linked by an edge in $G$ if and only if the images $f\left(v_{0}\right)$ and $f\left(v_{1}\right)$ are linked by an edge in $G^{\prime}$ ). Similarly, a full subgraph of $G^{\prime}$ is a graph of the form $G=\left.G^{\prime}\right|_{V}=\left(V, E^{\prime} \cap V^{2}\right)$ for some subset $V \subseteq V^{\prime}$, so the inclusion map gives a full embedding $G \rightarrow G^{\prime}$.

Lemma 10.3. Suppose we have a finite graph $G^{\prime}$, a full subgraph $G$, and a full embedding $f: G \rightarrow \Gamma$. Then there is a full embedding $f^{\prime}: G^{\prime} \rightarrow \Gamma$ extending $f$.

Proof. It is easy to reduce to the case where $G^{\prime}$ has only one more vertex than $G$, say $V^{\prime}=V \amalg\{x\}$. Put $A=\left\{v \in V \mid(v, x) \in E^{\prime}\right\}$ and $N=\max \{f(v) \mid v \in V\}+1$, then let $f^{\prime}: V^{\prime} \rightarrow \mathbb{N}$ be the map extending $f$ with $f^{\prime}(x)=2^{N}+\sum_{v \in A} 2^{f(v)}$. It is straightforward to check that this has the required properties.

Remark 10.4. As we mentioned in Example 4.7, each group $E_{k}$ (for $k \geq 0$ ) is isomorphic to $\mathbb{F}$. The generator is the element $y_{k}=x_{B(k)}=\prod_{i \in B(k)} x_{i}$. We say that a finite subset $I \subseteq \mathbb{N}$ is $\Gamma$-complete if the full subgraph $\left.\Gamma\right|_{I}$ is a complete graph (so 
every two distinct points are linked by an edge). We say that a natural number $n$ is $B \Gamma$-complete if $B(n)$ is $\Gamma$-complete. It is clear that the set

$$
\left\{y_{n} \mid n \text { is not } B \Gamma \text {-complete }\right\}
$$

is a basis for the Rado ideal, and thus that the set

$$
\left\{y_{n} \mid n \text { is } B \Gamma \text {-complete }\right\}
$$

gives a basis for $Q$.

Proposition 10.5. For any finitely generated ideal $I \leq Q$, we have $\operatorname{ann}^{2}(I)=I$. (In other words, $Q$ satisfies the double annihilator condition.)

Proof. Let $I \leq Q$ be a finitely generated ideal. Because of Remark 10.4, the ideal $I$ must be generated by a finite list of monomials, say $I=\left(x_{A_{1}}, \ldots, x_{A_{r}}\right)$, where each $A_{i}$ is a finite $\Gamma$-complete subset of $\mathbb{N}$. Similarly, $\operatorname{ann}^{2}(I)$ is generated by the monomials that it contains.

Let $T$ be another $\Gamma$-complete subset of $\mathbb{N}$. If $T$ contains $A_{i}$ for some $i$, it is clear that $x_{T} \in I$. Suppose instead that $T$ does not contain any of the $A_{i}$. Let $N$ be strictly larger than any of the elements of $T \cup \bigcup_{i} A_{i}$, and put $n=2^{N}+\sum_{t \in T} 2^{t}$, so $B(n)=\{N\} \cup T$. It is clear that $n \notin T$ and $T \cup\{n\}$ is $\Gamma$-complete so $x_{n} x_{T} \neq 0$. However, we claim that $x_{n} x_{A_{i}}=0$ for all $i$. Indeed, as $T \nsupseteq A_{i}$ we can choose $k \in A_{i} \backslash T$. As $N$ is so large we cannot have $n \in B(k)$, and also $k \notin\{N\} \cup T=B(n)$, so $x_{n} x_{k}=0$, and so $x_{n} x_{A_{i}}=0$ as claimed. We now see that $x_{n} \in \operatorname{ann}(I)$, but $x_{n} x_{T} \neq 0$, so $x_{T} \notin \operatorname{ann}^{2}(I)$. It follows that $\operatorname{ann}^{2}(I)=I$ as claimed.

Proposition 10.6. $Q$ is not self-injective.

Proof. Take any pair $p, q \in \mathbb{N}$ with $p \neq q$ and $x_{p} x_{q}=0$ (say $p=0$ and $q=2$ ). Put $u=\left(x_{p}, x_{q}\right)$ and $v=\left(0, x_{q}\right)$, and consider the test pair $(u, v)$. Any transporter would have to be an element $t \in Q_{0}=\{0,1\}$ with $t x_{p}=0$ and $t x_{q}=x_{q}$. It is clear from this that there is no transporter. A block would be a pair $(a, b)$ with $b x_{q} \neq 0$ but $a x_{p}+b x_{q}=0$ (so $a x_{p}=b x_{q} \neq 0$ ). This means that $a$ and $b$ are nonzero homogeneous elements, say $a=x_{A}$ and $b=x_{B}$ for some $\Gamma$-complete sets $A$ and $B$. As $a x_{p} \neq 0$ we see that $p \notin A$, and that $A \cup\{p\}$ is again $\Gamma$-complete. Similarly, we have $q \notin B$ and $B \cup\{q\}$ is $\Gamma$-complete. The equation $a x_{p}=b x_{q}$ means that $A \cup\{p\}=B \cup\{q\}$, so we have $A=C \cup\{q\}$ and $B=C \cup\{p\}$ for some set $C$. This now gives $b x_{q}=x_{C} x_{p} x_{q}$, but $x_{p} x_{q}=0$ so $b x_{q}=0$, contrary to assumption. This shows that we have neither a block nor a transporter, so $Q$ is not self-injective.

Remark 10.7. We could give $Q$ a different grading such that there are some pairs $(i, j)$ with $i \neq j$ but $\left|x_{i}\right|=\left|x_{j}\right|$, so $x_{i}+x_{j}$ becomes homogeneous. One can check that if $x_{i} x_{j}=0$ then $\operatorname{ann}^{2}\left(x_{i}+x_{j}\right)=\left(x_{i}, x_{j}\right) \neq\left(x_{i}+x_{j}\right)$, so the double annihilator condition no longer holds. We will discuss a similar situation with more details in 
Lemma 11.18. We believe that the self-injectivity condition is similarly sensitive to the choice of grading, but we do not have an example to prove this.

Proposition 10.8. $Q$ is totally incoherent.

Proof. First, it is clear that $Q$ is local, with maximal ideal $\mathfrak{m}=\left(x_{i} \mid i \in \mathbb{N}\right)=$ $\bigoplus_{k>0} Q_{k}$. The generators $x_{i}$ form a basis for $\mathfrak{m} / \mathfrak{m}^{2}$. Note that if $A \subset \mathbb{N}$ is nonempty and $\Gamma$-complete, then infinitely many of the variables $x_{i}$ will satisfy $x_{i} x_{A}=0$, so the image of $\operatorname{ann}\left(x_{A}\right)$ in $\mathfrak{m} / \mathfrak{m}^{2}$ will have infinite dimension. The claim therefore follows by Corollary 5.6.

\section{The $\epsilon_{0}$-algebra}

The $\epsilon_{0}$-algebra $A$ was introduced in Definition 1.19. We now explain the definition in more detail, and prove some properties.

Definition 11.1. Suppose we have a sequence $\underline{\beta}=\left(\beta_{1}>\beta_{2}>\cdots>\beta_{r}\right)$ of ordinals, and a sequence $\underline{n}=\left(n_{1}, \ldots, n_{r}\right)$ of positive integers. We write

$$
C(\underline{\beta}, \underline{n})=\omega^{\beta_{1}} n_{1}+\cdots+\omega^{\beta_{r}} n_{r} .
$$

Note that this uses ordinal exponentiation, defined in the usual recursive way by $\alpha^{\beta+1}=\alpha \alpha^{\beta}$ and $\alpha^{\lambda}=\bigcup_{\beta<\lambda} \alpha^{\beta}$ when $\lambda$ is a limit ordinal.

The following fact is standard (and not hard to prove by transfinite induction).

Proposition 11.2. For any ordinal $\alpha$, there is a unique pair $(\underline{\beta}, \underline{n})$ such that $\alpha=C(\underline{\beta}, \underline{n})$. (This is the Cantor normal form for $\alpha$.)

Proof. See [Johnstone 1987, Exercise 6.10], for example.

Definition 11.3. We put $\pi_{0}=\omega$ and define $\pi_{n}$ recursively by $\pi_{n+1}=\omega^{\pi_{n}}$, and then put $\epsilon_{0}=\bigcup_{n} \pi_{n}$.

One can check that $\epsilon_{0}=\omega^{\epsilon_{0}}$, and that $\epsilon_{0}$ is the smallest ordinal with this property. Note that the expression $\epsilon_{0}=\omega^{\epsilon_{0}}$ is the Cantor normal form of $\epsilon_{0}$. For $\alpha<\epsilon_{0}$ we find that the exponents $\beta_{t}$ in the Cantor normal form of $\alpha$ are strictly less than $\alpha$, so in this case one can do induction or recursion based on the Cantor normal form.

Definition 11.4. We define $\delta: \epsilon_{0} \rightarrow \mathbb{N}$ recursively by $\delta(0)=1$, and $\delta(\alpha)=$ $\left(\sum_{t}\left(\delta\left(\beta_{t}\right)+2\right) n_{t}\right)-1$ if $\alpha=\omega^{\beta_{1}} n_{1}+\cdots+\omega^{\beta_{r}} n_{r}$.

We will give enough examples to show that $\delta$ is not injective, which will be needed later. 


\section{Example 11.5.}

$$
\begin{aligned}
\delta(1) & =\delta\left(\omega^{0}\right)=(\delta(0)+2)-1=2, \\
\delta(2) & =\delta\left(\omega^{0} 2\right)=(\delta(0)+2) 2-1=5, \\
\delta(\omega) & =\delta\left(\omega^{1}\right)=(\delta(1)+2)-1=3, \\
\delta(\omega+1) & =\delta\left(\omega^{1}+\omega^{0}\right)=(\delta(1)+2)+(\delta(0)+2)-1=6, \\
\delta\left(\omega^{2}\right) & =(\delta(2)+2)-1=6 .
\end{aligned}
$$

In order to analyse $\delta$, it is helpful to modify the Cantor normal form slightly.

Lemma 11.6. If $\alpha<\epsilon_{0}$ then there is a unique way to write

$$
\alpha=\omega^{\beta_{1}}+\omega^{\beta_{2}}+\cdots+\omega^{\beta_{m}}
$$

with $\alpha>\beta_{1} \geq \beta_{2} \geq \cdots \geq \beta_{m}$. (This is the expanded Cantor normal form.)

Proof. Just take the ordinary Cantor normal form and replace $\omega^{\beta_{t}} n_{t}$ by $n_{t}$ copies of $\omega^{\beta_{t}}$.

Lemma 11.7. For any $d \in \mathbb{N}$ there are only finitely many ordinals $\alpha \in \epsilon_{0}$ with $\delta(\alpha)=d$.

Proof. Let $A$ denote the alphabet $\{0, \pi,+\}$. For each $\alpha<\epsilon_{0}$ we define a word $\phi(\alpha)$ in $A$ as follows. We start with $\phi(0)=0$. If $\theta>0$ has expanded Cantor normal form $\theta=\omega^{\beta_{1}}+\cdots+\omega^{\beta_{m}}$ we put

$$
\phi(\theta)=\phi\left(\beta_{1}\right) \pi \phi\left(\beta_{2}\right) \pi \cdots \phi\left(\beta_{m}\right) \pi+\cdots+
$$

(with $m-1$ plusses at the end). For example we have

$$
\begin{aligned}
& \phi(3)=\phi\left(\omega^{0}+\omega^{0}+\omega^{0}\right)=0 \pi 0 \pi 0 \pi++, \\
& \phi\left(\omega^{\omega}+\omega\right)=0 \pi \pi \pi 0 \pi \pi+.
\end{aligned}
$$

It is clear from the definitions that $\delta(\theta)$ is the length of $\phi(\theta)$, and there are only $3^{d}$ words in $A$ of length $d$, so it will suffice to show that $\phi$ is injective. If we interpret $\pi$ as the operator $x \mapsto \omega^{x}$ then $\phi(\theta)$ is a reverse Polish expression that evaluates to $\theta$, and this implies injectivity.

Corollary 11.8. $\epsilon_{0}$ is countable.

Definition 11.9. Let $\tilde{A}$ be the graded polynomial algebra over $\mathbb{F}$ generated by elements $x_{\alpha}$ for each ordinal $\alpha<\epsilon_{0}$, with $\left|x_{\alpha}\right|=\delta(\alpha)$.

Using Lemma 11.7 we see that $\tilde{A}_{d}$ is finite for all $d$. 
Definition 11.10. For ordinals $\alpha, \beta<\epsilon_{0}$ with $\alpha \neq \beta$ we define $\mu_{0}(\alpha, \beta)$ to be the coefficient of $\omega^{\beta}$ in $\alpha$. More explicitly, if the Cantor normal form of $\alpha$ involves a term $\omega^{\beta} n$, then $\mu_{0}(\alpha, \beta)=n$; if there is no such term then $\mu_{0}(\alpha, \beta)=0$. One can check that if $\mu_{0}(\alpha, \beta)>0$ then $\mu_{0}(\beta, \alpha)=0$. We put $\mu(\alpha, \beta)=\max \left(\mu_{0}(\alpha, \beta), \mu_{0}(\beta, \alpha)\right)$.

Proposition 11.11. For any finite set $J \subset \epsilon_{0}$ and map $v: J \rightarrow \mathbb{N}$ there exists $\alpha \in \epsilon_{0} \backslash J$ such that $\mu(\alpha, \beta)=v(\beta)$ for all $\beta \in J$. (We will call this the extension property.)

Proof. Write $J$ in order as $J=\left\{\beta_{1}>\beta_{2}>\cdots>\beta_{r}\right\}$ and then take

$$
\alpha=\omega^{\beta_{1}+1}+\omega^{\beta_{1}} \cdot v\left(\beta_{1}\right)+\cdots+\omega^{\beta_{r}} \cdot v\left(\beta_{r}\right) .
$$

It is visible that $\mu_{0}\left(\alpha, \beta_{t}\right)=v\left(\beta_{t}\right)$ for all $t$. Also, because of the initial term $\omega^{\beta_{1}+1}$ we have $\omega^{\alpha}>\alpha>\beta_{t}$ for all $t$ and so $\mu_{0}\left(\beta_{t}, \alpha\right)=0$. It follows that $\mu\left(\alpha, \beta_{t}\right)=v\left(\beta_{t}\right)$ for all $t$, as required.

From now on we will only need the fact that our index set $\epsilon_{0}$ is countable and that the extension property holds. It will therefore be notationally convenient to write $I=\epsilon_{0}$ and ignore the fact that the elements of $I$ are ordinals, and to write $i$ instead of $\alpha$ for a typical element of $I$. We also put $I_{2}=\left\{(i, j) \in I^{2} \mid i \neq j\right\}$.

Definition 11.12. For each $(i, j) \in I_{2}$ we put $\rho(i, j)=x_{i} x_{j}^{\mu(i, j)+1}$. We then let $A$ be the quotient of $\tilde{A}$ by all such elements $\rho(i, j)$. We call this the $\epsilon_{0}$-algebra.

Definition 11.13. Given a map $\alpha: I \rightarrow \mathbb{N}$, we write $\operatorname{supp}(\alpha)=\{i \mid \alpha(i)>0\}$. Let $M \tilde{A}$ be the set of all such maps $\alpha$ for which $\operatorname{supp}(\alpha)$ is finite. For $\alpha \in M \tilde{A}$ we put $x^{\alpha}=\prod_{i} x_{i}^{\alpha(i)} \in \tilde{A}$. We write $B \tilde{A}$ for the set of all such monomials $x^{\alpha}$, so $B \tilde{A}$ is a basis for $\tilde{A}$. Next, put

$$
M A=\{\alpha \in M \tilde{A} \mid \forall i \neq j \alpha(i)>0 \Rightarrow \alpha(j) \leq \mu(i, j)\}
$$

and $B A=\left\{x^{\alpha} \mid \alpha \in M A\right\}$. One can check that $B A$ gives a basis for $A$.

Definition 11.14. A monomial ideal is just an ideal in $A$ that is generated by some subset of $B A$.

Remark 11.15. Let $P$ be a monomial ideal, generated by $\left\{x^{\alpha} \mid \alpha \in U\right\}$ for some subset $U \subseteq M A$. Put

$$
U^{+}=\{\alpha \in M A \mid \alpha \geq \beta \text { for some } \beta \in U\} .
$$

It is easy to see that $\left\{x^{\alpha} \mid \alpha \in U^{+}\right\}$is then a basis for $P$ over $\mathbb{F}$. It follows easily that sums, products, intersections and annihilators of monomial ideals are again monomial ideals. 
Lemma 11.16. If $P$ is a monomial ideal then it is finitely generated if and only if there is a finite list of monomials that generate it.

Proof. Suppose that $P$ is generated by $a_{1}, \ldots, a_{m}$, where the elements $a_{t}$ need not be monomials. We can write $a_{t}=\sum_{\alpha \in U_{t}} a_{t, \alpha} x^{\alpha}$, for some finite set $U_{t} \subset M A$ and some nonzero coefficients $a_{t, \alpha}$. Using Remark 11.15 we see that the terms $x^{\alpha}$ (for $\left.\alpha \in U_{t}\right)$ lie in $P$. Put $U=\bigcup_{t} U_{t}$ (which is finite) and put $P^{\prime}=\left(x^{\alpha} \mid \alpha \in U\right) \leq P$. Clearly $a_{t} \in\left(x^{\alpha} \mid \alpha \in U_{t}\right) \leq P^{\prime}$ and the elements $a_{t}$ generate $P$ so $P \leq P^{\prime}$ and so $P=P^{\prime}$. Thus, $P$ is generated by a finite list of monomials.

Proposition 11.17. Let $P \leq A$ be a finitely generated monomial ideal. Then $\operatorname{ann}^{2}(P)=P$.

Proof. It is automatic that $P \leq \operatorname{ann}^{2}(P)$, so it will suffice to prove the opposite inclusion. Note that both $P$ and $\operatorname{ann}^{2}(P)$ are monomial ideals, so it will suffice to show that they contain the same monomials. Suppose that $x^{\beta}$ is a nonzero monomial that does not lie in $P$; we must find $y \in \operatorname{ann}(P)$ such that $x^{\beta} y \neq 0$.

We can choose a finite list $\alpha_{1}, \ldots, \alpha_{r} \in M$ such that $P=\left(x^{\alpha_{1}}, \ldots, x^{\alpha_{r}}\right)$. Put $J=\operatorname{supp}(\beta) \cup \bigcup_{i} \operatorname{supp}\left(\alpha_{i}\right)$, which is a finite subset of $I$. Put $N=\max \{\beta(j) \mid j \in J\}$.

Next, for each $t$ we note that $x^{\beta}$ cannot be divisible by $x^{\alpha_{t}}$, so we can choose $i_{t} \in J$ such that $\alpha_{t}\left(i_{t}\right)>\beta\left(i_{t}\right)$. Using the extension property we can recursively define distinct elements $k_{1}, \ldots, k_{r} \in I \backslash J$ such that

(a) $\mu\left(k_{t}, i_{t}\right)=\alpha_{t}\left(i_{t}\right)-1$,

(b) $\mu\left(k_{t}, j\right)=N$ for $j \in J \backslash\left\{i_{t}\right\}$,

(c) $\mu\left(k_{t}, k_{s}\right)=1$ for $s<t$.

Put $y=\prod_{t} x_{k_{t}}$. This is nonzero by property (c). Property (a) tells us that $x_{j_{t}} x^{\alpha_{t}}=0$ for all $t$, which implies that $y \in \operatorname{ann}(A)$. On the other hand:

- Clause (a) above tells us that $y x^{\beta}$ is not divisible by any relator $\rho\left(k_{t}, i_{t}\right)$.

- Clause (b) tells us that $y x^{\beta}$ is not divisible by any relator $\rho\left(k_{t}, j\right)$ with $j \in$ $J \backslash\left\{i_{t}\right\}$.

- Clause (c) tells us that $y x^{\beta}$ is not divisible by any relator $\rho\left(k_{t}, k_{s}\right)$.

- Our original assumption $x^{\beta} \neq 0$ implies that $y x^{\beta}$ is not divisible by any relator $\rho\left(j, j^{\prime}\right)$ with $j, j^{\prime} \in J$.

This shows that $y x^{\beta} \neq 0$, but $y \in \operatorname{ann}(P)$, so $x^{\beta} \notin \operatorname{ann}^{2}(P)$, as claimed.

Lemma 11.18. Let $i$ and $j$ be any two distinct indices in $I$ with $\left|x_{i}\right|=\left|x_{j}\right|$ and $\mu(i, j)=0$. Then $\operatorname{ann}^{2}\left(x_{i}+x_{j}\right)=\left(x_{i}, x_{j}\right)>\left(x_{i}+x_{j}\right)$.

Proof. As $\mu(i, j)=0$ we have $x_{i} x_{j}=0$ and so (using monomial bases) $\left(x_{i}\right) \cap\left(x_{j}\right)=0$. If $u\left(x_{i}+x_{j}\right)=0$ then we have $u x_{i}=-u x_{j}$, with the left hand side in $\left(x_{i}\right)$ and the right hand side in $\left(x_{j}\right)$. As $\left(x_{i}\right) \cap\left(x_{j}\right)=0$ this gives $u x_{i}=u x_{j}=0$. It now follows 
that $\operatorname{ann}\left(x_{i}+x_{j}\right)=\operatorname{ann}\left(x_{i}, x_{j}\right)$, and so $\operatorname{ann}^{2}\left(x_{i}+x_{j}\right)=\operatorname{ann}^{2}\left(x_{i}, x_{j}\right)$. As $\left(x_{i}, x_{j}\right)$ is a monomial ideal we also have $\operatorname{ann}^{2}\left(x_{i}, x_{j}\right)=\left(x_{i}, x_{j}\right)$, so $\operatorname{ann}^{2}\left(x_{i}+x_{j}\right)=\left(x_{i}, x_{j}\right)>$ $\left(x_{i}+x_{j}\right)$ as claimed.

Corollary 11.19. Example 11.5 shows that the lemma applies to the pair $\left(\omega^{2}, \omega+1\right)$, so A does not satisfy the double annihilator condition. Thus, Remark 2.4 shows that A cannot be self-injective.

Remark 11.20. We could choose a different grading such that all the generators had different degrees, which would eliminate any examples as in Lemma 11.18. However, we cannot ensure that $A_{d}$ has dimension at most one for all $d$, because when $i \neq j$ the elements $x_{i}^{\left|x_{j}\right|}$ and $x_{j}^{\left|x_{i}\right|}$ have the same degree and are linearly independent. Thus, there will always be ideals that are not monomial ideals. We suspect that there is no grading for which $A$ satisfies the full double annihilator condition, but we have not proved this.

Proposition 11.21. A is totally incoherent.

Proof. Put $\mathfrak{m}_{0}=0$ and $\mathfrak{m}_{k}=A_{k}$ for all $k>0$, so $A / \mathfrak{m}=\mathbb{F}$. It is clear that $\mathfrak{m}$ is an ideal, and that the (homogeneous) elements of $\mathfrak{m}$ are precisely the elements of $A$ that are not invertible. Given this, it follows that $\mathfrak{m}$ is the unique maximal ideal in $A$, so $A$ is local. From the form of the relations in $A$ we see that $\left\{x_{i} \mid i \in I\right\}$ is a basis for $\mathfrak{m} / \mathfrak{m}^{2}$.

Now consider an element $a \in A_{d}$ for some $d>0$. Put

$$
\begin{aligned}
& U=\{i \in I \mid \delta(i) \leq d\}, \\
& V=\left\{\omega^{i} \mid i \in I \backslash U\right\} .
\end{aligned}
$$

We find that $x_{i} x_{j}=0$ for all $i \in U$ and $j \in V$. Moreover, we have $a \in\left(x_{i} \mid i \in U\right)$, so $a x_{j}=0$ for all $j \in V$, so the image of $\operatorname{ann}(a)$ in $\mathfrak{m} / \mathfrak{m}^{2}$ has infinite dimension.

Now let $P$ be a finitely presented ideal in $A$. If $P=\mathfrak{m} P$ then $P=0$ by Nakayama's lemma. Otherwise, we can choose $a \in P \backslash \mathfrak{m} P$, and Lemma 5.5 tells us that ann $(a)$ has finite image in $\mathfrak{m} / \mathfrak{m}^{2}$. The above remarks show that we must have $|a|=0$, and $a \notin \mathfrak{m} P$ so $a \neq 0$. Thus $a$ is invertible, so $P=A$.

Proposition 11.22. The reduced quotient is

$$
A / \sqrt{0}=\mathbb{F}\left[x_{i} \mid i \in I\right] /\left(x_{i} x_{j} \mid i \neq j\right) .
$$

Proof. In $A$ we have $x_{i} x_{j}^{\mu(i, j)+1}=0$, so $\left(x_{i} x_{j}\right)^{\mu(i, j)+1}=0$, and so $x_{i} x_{j}$ is nilpotent. If we put

$$
A^{\prime}=A /\left(x_{i} x_{j} \mid i \neq j\right)=\mathbb{F}\left[x_{i} \mid i \in I\right] /\left(x_{i} x_{j} \mid i \neq j\right),
$$

we deduce that $A / \sqrt{0}=A^{\prime} / \sqrt{0}$. However, it is easy to see that $A^{\prime}$ is already reduced, so $A / \sqrt{0}=A^{\prime}$ as claimed. 


\section{Triangulation}

Recall that a triangulated category is a triple $(\mathscr{C}, \Sigma, \Delta)$, where $\mathscr{C}$ is an additive category, and $\Sigma: \mathscr{b} \rightarrow \mathscr{C}$ is an equivalence, and $\Delta$ is a class of diagrams of shape

$$
X \rightarrow Y \rightarrow Z \rightarrow \Sigma X
$$

(called distinguished triangles), subject to certain axioms that we will not list here.

Definition 12.1. Let $R$ be a self-injective graded ring, let $\operatorname{Mod}_{R}$ be the category of $R$-modules, and let $\Sigma: \operatorname{Mod}_{R} \rightarrow \operatorname{Mod}_{R}$ be the usual suspension functor so that $(\Sigma M)_{i}=M_{i-1}$. Let $\operatorname{InjMod}_{R}$ be the full subcategory of injective modules. A triangulation structure for $R$ is a pair $(\mathcal{N}, \Delta)$, where:

(a) $\mathcal{N}$ is a full subcategory of $\operatorname{Inj} \operatorname{Mod}_{R}$ containing $R$.

(b) $\mathcal{N}$ is closed under finite direct sums, retracts, suspensions and desuspensions.

(c) $\Delta$ is a class of distinguished triangles making $(\mathcal{N}, \Sigma, \Delta)$ into a triangulated category.

We can also make a similar definition for ungraded rings.

Definition 12.2. Let $R$ be a self-injective ungraded ring. An ungraded triangulation structure for $R$ is a pair $(\mathcal{N}, \Delta)$, where:

(a) $\mathcal{N}$ is a full subcategory of $\operatorname{Inj} \operatorname{Mod}_{R}$ containing $R$.

(b) $\mathcal{N}$ is closed under finite direct sums, retracts, suspensions and desuspensions.

(c) $\Delta$ is a class of distinguished triangles making $(\mathcal{N}, 1, \Delta)$ into a triangulated category.

In [Muro et al. 2007] we constructed ungraded triangulation structures for $\mathbb{Z} / 4$ and for $K[\epsilon] / \epsilon^{2}$ (where $K$ is any field of characteristic two). If Freyd's generating hypothesis is true, then the image of the functor $\pi_{*}$ gives a graded triangulation structure for the ring $\pi_{*}(S)_{p}^{\wedge}$. We have not succeeded in constructing any examples of graded triangulation structures by pure algebra. Here we offer only some rather limited and negative results.

Lemma 12.3. If $(\mathcal{N}, \Delta)$ is a triangulation structure (in the graded or ungraded context) then all distinguished triangles in $\Delta$ are exact sequences.

Proof. The general theory of triangulated categories tells us that all functors of the form $\mathcal{N}(X,-)$ send distinguished triangles to long exact sequences. By assumption we have $R \in \mathcal{N}$, and we can take $X=R$ to prove the claim.

Lemma 12.4. If $(\mathcal{N}, \Delta)$ is a triangulation structure then all surjective maps in $\mathcal{N}$ are split. 
Proof. Let $M \stackrel{f}{\rightarrow} N$ be a surjective map in $\mathcal{N}$. This must fit into a distinguished triangle $L \stackrel{e}{\rightarrow} M \stackrel{f}{\rightarrow} N \stackrel{g}{\rightarrow} \Sigma L$. Here $g f=0$, but $f$ is surjective so $g=0$. It is standard that the functor $\mathcal{N}(N,-)$ converts our distinguished triangle to an exact sequence, so $f_{*}: \mathcal{N}(N, M) \rightarrow \mathcal{N}(N, N)$ is surjective. We can thus find $h: N \rightarrow M$ with $f h=1$, so $h$ splits $f$.

Corollary 12.5. If $(\mathcal{N}, \Delta)$ is a triangulation structure then all finitely generated modules in $\mathcal{N}$ are projective. Thus, if $R$ is local then all such modules are free.

Proof. Let $N$ be a finitely generated module in $\mathcal{N}$. This means that there is a surjective homomorphism $f: F \rightarrow N$ for some finitely generated free module $F$. As $\mathcal{N}$ is standard we see that $F \in \mathcal{N}$, so the lemma tells us that $N$ is a retract of $F$, so it is projective. It is well-known that finitely generated projective modules over local rings are free.

Proposition 12.6. Suppose that $R$ is a local graded ring with $R_{i}=0$ for $i<0$, and suppose that $R$ admits a triangulation structure. Then $R$ is totally incoherent.

Proof. Let $\mathfrak{m}$ be the unique maximal ideal, and let $(\mathcal{N}, \Delta)$ be a triangulation structure. It is not hard to see that $\mathfrak{m}_{0}$ is the unique maximal ideal in $R_{0}$, so $R_{0}$ is a local ring in the ungraded sense.

Let $J$ be any finitely generated ideal. We can then find a finitely generated free module $Q$ and an epimorphism $Q \rightarrow J$ such that $Q / \mathfrak{m} Q \rightarrow J / \mathfrak{m} J$ is an isomorphism. We will write $g$ for the composite map $Q \rightarrow J \rightarrow R$, so that $J=\operatorname{image}(g)$. If $J$ is finitely presented then $\operatorname{ker}(g)$ is again finitely generated, so we can find a finitely generated free module $P$ and a map $f: P \rightarrow Q$ with image $(f)=\operatorname{ker}(g)$ and $P / \mathfrak{m} P \stackrel{\widetilde{ }}{\rightarrow} \operatorname{ker}(g) / \mathfrak{m} \operatorname{ker}(g)$. With these minimal choices for $P$ and $Q$, it is clear that $P_{i}=Q_{i}=0$ when $i<0$. Next, we can fit $g$ into a distinguished triangle $\Sigma^{-1} R \stackrel{d}{\rightarrow} K \stackrel{i}{\rightarrow} Q \stackrel{g}{\rightarrow} R$. As $g f=0$, we can find a lift $\tilde{f}: P \rightarrow K$ with $i \tilde{f}=f$. We can combine this with $d$ to give a map $P \oplus \Sigma^{-1} R \rightarrow K$, and a diagram chase shows that this is surjective. Using Lemma 12.4 we deduce that this map is split epi and that $K$ is a finitely generated free module. It follows that $K_{i}=0$ for $i<-1$ and that $K_{-1}$ is a retract of $R_{0}$. As $R_{0}$ is local we must have either $K_{-1}=0$ or $K_{-1}=R_{0}$. If $K_{-1}=0$ then $d: \Sigma^{-1} R \rightarrow K$ must be zero, which implies that $g: Q \rightarrow R$ is split epi, which means that $J=R$. If $K_{-1} \neq 0$ then we find that $d$ must induce a monomorphism $\Sigma^{-1} R / \mathfrak{m} \rightarrow K$, and as $R$ is local this implies that $d$ is a split monomorphism, and thus that $g=0$ and so $J=0$.

Remark 12.7. As mentioned previously, there is an ungraded triangulation structure for the ring $\mathbb{Z} / 4$. The ideal $(2)<\mathbb{Z} / 4$ is finitely presented and is neither 0 nor $\mathbb{Z} / 4$. It follows that our grading assumptions are playing an essential role in the proof of the above proposition. 
Corollary 12.8. Neither the infinite exterior algebra (as in Example 4.7) nor the cube algebra (as in Section 7) admits a triangulation structure.

Proof. Both rings are coherent, by Propositions 5.4 and 7.25.

\section{References}

[Baer 1940] R. Baer, "Abelian groups that are direct summands of every containing abelian group", Bull. Amer. Math. Soc. 46 (1940), 800-806. MR 2,126i Zbl 0024.14902

[Benson et al. 2007] D. J. Benson, S. K. Chebolu, J. D. Christensen, and J. Mináč, "The generating hypothesis for the stable module category of a p-group", J. Algebra 310:1 (2007), 428-433. MR 2007k:16011 Zbl 1120.20002

[Bruns and Herzog 1993] W. Bruns and J. Herzog, Cohen-Macaulay rings, Cambridge Studies in Advanced Mathematics 39, Cambridge University Press, 1993. MR 95h:13020 Zbl 0788.13005

[Cameron 2001] P. J. Cameron, “The random graph revisited”, pp. 267-274 in European Congress of Mathematics (Barcelona, 2000), vol. 1, edited by C. Casacuberta et al., Progr. Math. 201, Birkhäuser, Basel, 2001. MR 2003j:05110 Zbl 1023.05124

[Carlson et al. 2009] J. F. Carlson, S. K. Chebolu, and J. Mináč, "Freyd's generating hypothesis with almost split sequences", Proc. Amer. Math. Soc. 137:8 (2009), 2575-2580. MR 2010c:20007 Zbl 1188.20006

[Devinatz 1990] E. S. Devinatz, “ $K$-theory and the generating hypothesis”, Amer. J. Math. 112:5 (1990), 787-804. MR 91i:55011 Zbl 0783.55008

[Freyd 1966] P. Freyd, "Stable homotopy", pp. 121-172 in Proceedings of the Conference on Categorical Algebra (La Jolla, CA, 1965), edited by S. Eilenberg et al., Springer, New York, 1966. MR 35 \#2280 Zbl 0195.52901

[Hovey 2007] M. Hovey, “On Freyd's generating hypothesis”, Q. J. Math. 58:1 (2007), 31-45. MR 2008b:55014 Zbl 1173.55007

[Hovey et al. 2007] M. Hovey, K. Lockridge, and G. Puninski, "The generating hypothesis in the derived category of a ring”, Math. Z. 256:4 (2007), 789-800. MR 2008b:18017 Zbl 1137.16016

[Johnstone 1987] P. T. Johnstone, Notes on logic and set theory, Cambridge University Press, 1987. MR 89e:03003 Zbl 0642.03001

[Lam 1999] T. Y. Lam, Lectures on modules and rings, Graduate Texts in Mathematics 189, Springer, New York, 1999. MR 99i:16001 Zbl 0911.16001

[Lockridge 2007] K. H. Lockridge, "The generating hypothesis in the derived category of $R$-modules", J. Pure Appl. Algebra 208:2 (2007), 485-495. MR 2007i:55009 Zbl 1112.55011

[Matsumura 1980] H. Matsumura, Commutative ring theory, Kyoritsu Shuppan, Tokyo, 1980. In Japanese; translated in Cambridge Studies in Advanced Mathematics 8, Cambridge University Press, 1986. MR 88h:13001 Zbl 0603.13001

[Muro et al. 2007] F. Muro, S. Schwede, and N. Strickland, "Triangulated categories without models", Invent. Math. 170:2 (2007), 231-241. MR 2008g:18016 Zbl 1125.18009

[Rado 1964] R. Rado, "Universal graphs and universal functions”, Acta Arith. 9 (1964), 331-340. MR 30 \#2488 Zbl 0139.17303

[Ravenel 1984] D. C. Ravenel, "Localization with respect to certain periodic homology theories", Amer. J. Math. 106:2 (1984), 351-414. MR 85k:55009 Zbl 0586.55003

[Ravenel 1992] D. C. Ravenel, Nilpotence and periodicity in stable homotopy theory, Annals of Mathematics Studies 128, Princeton University Press, 1992. MR 94b:55015 Zbl 0774.55001 
Communicated by David Benson

Received 2012-06-06 Revised 2013-06-29 Accepted 2013-07-30

Ishepperson@rm.com

Dorset Software, 10 the Linnets, Cottenham,

Cambridge CB248XZ, United Kingdom

n.p.strickland@sheffield.ac.uk School of Mathematics and Statistics, University of Sheffield, The Hicks Building, Hounsfield Road, Sheffield S37RH,

United Kingdom 


\section{Algebra \& Number Theory}

msp.org/ant

\section{EDITORS}

MANAGING EDITOR

Bjorn Poonen

Massachusetts Institute of Technology

Cambridge, USA

\author{
EDITORIAL BOARD CHAIR \\ David Eisenbud \\ University of California \\ Berkeley, USA
}

\section{BOARD OF EDITORS}

Georgia Benkart

Dave Benson

Richard E. Borcherds

John H. Coates

J-L. Colliot-Thélène

Brian D. Conrad

Hélène Esnault

Hubert Flenner

Edward Frenkel

Andrew Granville

Joseph Gubeladze

Roger Heath-Brown

Ehud Hrushovski

Craig Huneke

Mikhail Kapranov

Yujiro Kawamata

János Kollár

Yuri Manin

Barry Mazur

Philippe Michel
University of Wisconsin, Madison, USA

University of Aberdeen, Scotland

University of California, Berkeley, USA

University of Cambridge, UK

CNRS, Université Paris-Sud, France

University of Michigan, USA

Freie Universität Berlin, Germany

Ruhr-Universität, Germany

University of California, Berkeley, USA

Université de Montréal, Canada

San Francisco State University, USA

Oxford University, UK

Hebrew University, Israel

University of Virginia, USA

Yale University, USA

University of Tokyo, Japan

Princeton University, USA

Northwestern University, USA

Harvard University, USA

École Polytechnique Fédérale de Lausanne
Susan Montgomery

Shigefumi Mori

Raman Parimala

Jonathan Pila

Victor Reiner

Karl Rubin

Peter Sarnak

Joseph H. Silverman

Michael Singer

Vasudevan Srinivas

J. Toby Stafford

Bernd Sturmfels

Richard Taylor

Ravi Vakil

Michel van den Bergh

Marie-France Vignéras

Kei-Ichi Watanabe

Efim Zelmanov

Shou-Wu Zhang
University of Southern California, USA

RIMS, Kyoto University, Japan

Emory University, USA

University of Oxford, UK

University of Minnesota, USA

University of California, Irvine, USA

Princeton University, USA

Brown University, USA

North Carolina State University, USA

Tata Inst. of Fund. Research, India

University of Michigan, USA

University of California, Berkeley, USA

Harvard University, USA

Stanford University, USA

Hasselt University, Belgium

Université Paris VII, France

Nihon University, Japan

University of California, San Diego, USA

Princeton University, USA

PRODUCTION

production@msp.org

Silvio Levy, Scientific Editor

See inside back cover or msp.org/ant for submission instructions.

The subscription price for 2014 is US $\$ 225 /$ year for the electronic version, and $\$ 400 /$ year $(+\$ 55$, if shipping outside the US) for print and electronic. Subscriptions, requests for back issues and changes of subscribers address should be sent to MSP.

Algebra \& Number Theory (ISSN 1944-7833 electronic, 1937-0652 printed) at Mathematical Sciences Publishers, 798 Evans Hall \#3840, c/o University of California, Berkeley, CA 94720-3840 is published continuously online. Periodical rate postage paid at Berkeley, CA 94704, and additional mailing offices.

ANT peer review and production are managed by EditFLOW ${ }^{\circledR}$ from Mathematical Sciences Publishers.

\section{PUBLISHED BY}

- mathematical sciences publishers

nonprofit scientific publishing

http://msp.org/

(C) 2014 Mathematical Sciences Publishers 


\section{Algebra \& Number Theory}

Volume $8 \quad$ No. $2 \quad 2014$

Large self-injective rings and the generating hypothesis

LEIGH SHEPPERSON and NEIL STRICKLAND

On lower ramification subgroups and canonical subgroups

303

SHIN HATTORI

Wild models of curves

DINO LORENZINI

Geometry of Wachspress surfaces

COREY IRVING and HAL SCHENCK

Daniel Goldstein, Robert M. GuRAlnick, Mark L. Lewis, AleXander Moretó, Gabriel Navarro and Pham HuU TieP

The homotopy category of injectives

AMNON NEEMAN

Essential dimension of spinor and Clifford groups

Vladimir Chernousov and AleXANDER MERKurJeV

On Deligne's category $\underline{\operatorname{Rep}}^{a b}\left(S_{d}\right)$

JONATHAN COMES and VICTOR OSTRIK

Algebraicity of the zeta function associated to a matrix over a free group algebra

CHRISTIAN KASSEL and Christophe REUTENAUER 